\title{
Introduction to Hybrid Materials
}

\author{
Guido Kickelbick
}

\section{1}

\section{Introduction}

Recent technological breakthroughs and the desire for new functions generate an enormous demand for novel materials. Many of the well-established materials, such as metals, ceramics or plastics cannot fulfill all technological desires for the various new applications. Scientists and engineers realized early on that mixtures of materials can show superior properties compared with their pure counterparts. One of the most successful examples is the group of composites which are formed by the incorporation of a basic structural material into a second substance, the matrix. Usually the systems incorporated are in the form of particles, whiskers, fibers, lamellae, or a mesh. Most of the resulting materials show improved mechanical properties and a well-known example is inorganic fiber-reinforced polymers. Nowadays they are regularly used for lightweight materials with advanced mechanical properties, for example in the construction of vehicles of all types or sports equipment. The structural building blocks in these materials which are incorporated into the matrix are predominantly inorganic in nature and show a size range from the lower micrometer to the millimeter range and therefore their heterogeneous composition is quite often visible to the eye. Soon it became evident that decreasing the size of the inorganic units to the same level as the organic building blocks could lead to more homogeneous materials that allow a further fine tuning of materials' properties on the molecular and nanoscale level, generating novel materials that either show characteristics in between the two original phases or even new properties. Both classes of materials reveal similarities and differences and an attempt to define the two classes will follow below. However, we should first realize that the origin of hybrid materials did not take place in a chemical laboratory but in nature.

\subsection{1}

\section{Natural Origins}

Many natural materials consist of inorganic and organic building blocks distributed on the (macro)molecular or nanoscale. In most cases the inorganic part 
provides mechanical strength and an overall structure to the natural objects while the organic part delivers bonding between the inorganic building blocks and/or the soft tissue. Typical examples of such materials are bone, or nacre.

The concepts of bonding and structure in such materials are intensively studied by many scientists to understand the fundamental processes of their formation and to transfer the ideas to artificial materials in a so-called biomimetic approach. The special circumstances under which biological hybrid inorganicorganic materials are formed, such as ambient temperatures, an aqueous environment, a neutral $\mathrm{pH}$ and the fascinating plethora of complex geometries produced under these conditions make the mimicking of such structures an ultimate goal for scientists. In particular the study of biomineralization and its shape control is an important target of many scientific studies. This primarily interface-controlled process still reveals many questions, in particular how such a remarkable level of morphological diversity with a multiplicity of functions can be produced by so few building blocks. In addition to questions concerning the composition of the materials, their unique structures motivate enquiry to get a deeper insight in their formation, often not only because of their beauty but also because of the various functions the structures perform. A complex hierarchical order of construction from the nanometer to the millimeter level is regularly found in nature, where every size level of the specific material has its function which benefits the whole performance of the material. Furthermore these different levels of complexity are reached by soft chemical self-assembly mechanisms over a large dimension, which is one of the major challenges of modern materials chemistry.

Chapter 7 describes the fundamental principles of biomineralization and hybrid inorganic-organic biomaterials and many applications to medical problems are shown in Chapter 8.

\section{1 .2}

\section{The Development of Hybrid Materials}

Although we do not know the original birth of hybrid materials exactly it is clear that the mixing of organic and inorganic components was carried out in ancient world. At that time the production of bright and colorful paints was the driving force to consistently try novel mixtures of dyes or inorganic pigments and other inorganic and organic components to form paints that were used thousands of years ago. Therefore, hybrid materials or even nanotechnology is not an invention of the last decade but was developed a long time ago. However, it was only at the end of the 20th and the beginning of the 21st century that it was realized by scientists, in particular because of the availability of novel physico-chemical characterization methods, the field of nanoscience opened many perspectives for approaches to new materials. The combination of different analytical techniques gives rise to novel insights into hybrid materials and makes it clear that bottomup strategies from the molecular level towards materials' design will lead to novel properties in this class of materials. 
Apart from the use of inorganic materials as fillers for organic polymers, such as rubber, it was a long time before much scientific activity was devoted to mixtures of inorganic and organic materials. One process changed this situation: the sol-gel process. This process, which will be discussed in more detail later on, was developed in the 1930s using silicon alkoxides as precursors from which silica was produced. In fact this process is similar to an organic polymerization starting from molecular precursors resulting in a bulk material. Contrary to many other procedures used in the production of inorganic materials this is one of the first processes where ambient conditions were applied to produce ceramics. The control over the preparation of multicomponent systems by a mild reaction method also led to industrial interest in that process. In particular the silicon based sol-gel process was one of the major driving forces what has become the broad field of inorganic-organic hybrid materials. The reason for the special role of silicon was its good processability and the stability of the $\mathrm{Si}-\mathrm{C}$ bond during the formation of a silica network which allowed the production of organic-modified inorganic networks in one step.

Inorganic-organic hybrids can be applied in many branches of materials chemistry because they are simple to process and are amenable to design on the molecular scale. Currently there are four major topics in the synthesis of inorganic-organic materials: (a) their molecular engineering, (b) their nanometer and micrometer-sized organization, (c) the transition from functional to multifunctional hybrids, and (d) their combination with bioactive components.

Some similarities to sol-gel chemistry are shown by the stable metal sols and colloids, such as gold colloids, developed hundreds of years ago. In fact sols prepared by the sol-gel process, i.e. the state of matter before gelation, and the gold colloids have in common that their building blocks are nanosized particles surrounded by a (solvent) matrix. Such metal colloids have been used for optical applications in nanocomposites for centuries. Glass, for example, was already colored with such colloids centuries ago. In particular many reports of the scientific examination of gold colloids, often prepared by reduction of gold salts, are known from the end of the 18th century. Probably the first nanocomposites were produced in the middle of the 19th century when gold salts were reduced in the presence of gum arabic. Currently many of the colloidal systems already known are being reinvestigated by modern instrumental techniques to get new insights into the origin of the specific chemistry and physics behind these materials.

\section{1 .3}

\section{Definition: Hybrid Materials and Nanocomposites}

The term hybrid material is used for many different systems spanning a wide area of different materials, such as crystalline highly ordered coordination polymers, amorphous sol-gel compounds, materials with and without interactions between the inorganic and organic units. Before the discussion of synthesis and properties of such materials we try to delimit this broadly-used term by taking into account various concepts of composition and structure (Table 1.1). The most wide-ranging 
Table 1.1 Different possibilities of composition and structure of hybrid materials.

Matrix:

Building blocks:

Interactions between components: crystalline $\leftrightarrow$ amorphous

organic $\leftrightarrow$ inorganic

molecules $\leftrightarrow$ macromolecules $\leftrightarrow$ particles $\leftrightarrow$ fibers strong $\leftrightarrow$ weak

definition is the following: a hybrid material is a material that includes two moieties blended on the molecular scale. Commonly one of these compounds is inorganic and the other one organic in nature. A more detailed definition distinguishes between the possible interactions connecting the inorganic and organic species. Class I hybrid materials are those that show weak interactions between the two phases, such as van der Waals, hydrogen bonding or weak electrostatic interactions. Class II hybrid materials are those that show strong chemical interations between the components. Because of the gradual change in the strength of chemical interactions it becomes clear that there is a steady transition between weak and strong interactions (Fig. 1.1). For example there are

Strength of interaction<smiles>[R][Si](C)(C)O[Si]12O[Si](C)(C)O[Si](C)(O[Si](C)(C)O1)O[Si](C)(C)O2</smiles>

\section{Covalent}

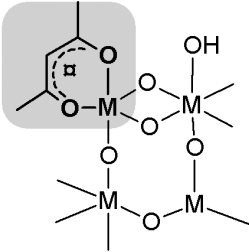

Coordinative

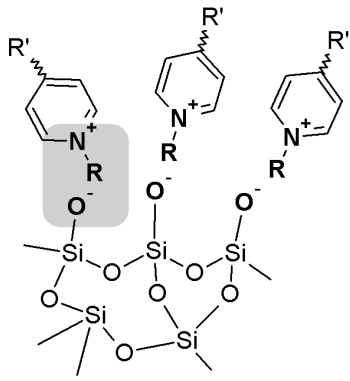

Ionic

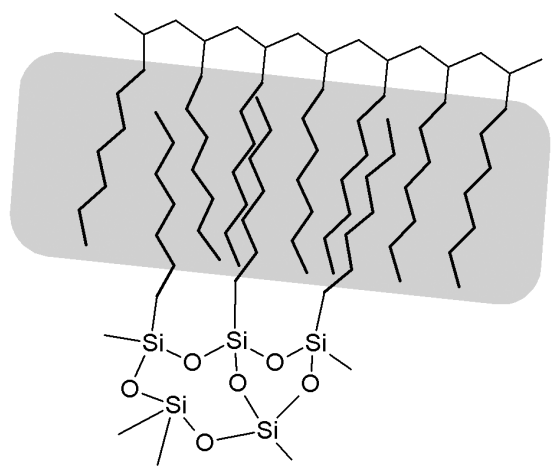

Van-der-Waals

Fig. 1.1 Selected interactions typically applied in hybrid materials and their relative strength. 
Table 1.2 Different chemical interactions and their respective strength.

\begin{tabular}{lcll}
\hline Type of interaction & Strength $\left[\mathrm{kJ} \mathrm{mol}^{-1}\right]$ & Range & Character \\
\hline van der Waals & ca. 50 & Short & $\begin{array}{c}\text { nonselective, } \\
\text { nondirectional } \\
\end{array}$ \\
H-bonding & $5-65$ & Short & selective, directional \\
Coordination bonding & $50-200$ & Short & directional \\
Ionic & $50-250^{[a]}$ & Long & nonselective \\
Covalent & 350 & Short & predominantly \\
& & & irreversible \\
\hline
\end{tabular}

a Depending on solvent and ion solution; data are for organic media.

hydrogen bonds that are definitely stronger than for example weak coordinative bonds. Table 1.2 presents the energetic categorization of different chemical interactions depending on their binding energies.

In addition to the bonding characteristics structural properties can also be used to distinguish between various hybrid materials. An organic moiety containing a functional group that allows the attachment to an inorganic network, e.g. a trialkoxysilane group, can act as a network modifying compound because in the final structure the inorganic network is only modified by the organic group. Phenyltrialkoxysilanes are an example for such compounds; they modify the silica network in the sol-gel process via the reaction of the trialkoxysilane group (Scheme 1.1a) without supplying additional functional groups intended to undergo further chemical reactions to the material formed. If a reactive functional group is incorporated the system is called a network functionalizer (Scheme 1.1c). The situation is different if two or three of such anchor groups modify an organic segment; this leads to materials in which the inorganic group is afterwards an integral part of the hybrid network (Scheme 1.1b). The latter systems are described in more detail in Chapter 6.

Blends are formed if no strong chemical interactions exist between the inorganic and organic building blocks. One example for such a material is the combination of inorganic clusters or particles with organic polymers lacking a strong (e.g. covalent) interaction between the components (Scheme 1.2a). In this case a material is formed that consists for example of an organic polymer with entrapped discrete inorganic moieties in which, depending on the functionalities of the components, for example weak crosslinking occurs by the entrapped inorganic units through physical interactions or the inorganic components are entrapped in a crosslinked polymer matrix. If an inorganic and an organic network interpenetrate each other without strong chemical interactions, so called interpenetrating networks (IPNs) are formed (Scheme 1.2b), which is for example the case if a sol-gel material is formed in presence of an organic polymer or vice versa. Both materials described belong to class I hybrids. Class II hybrids are formed when the discrete inorganic building blocks, e.g. clusters, are covalently bonded to the 
a) Network Modifier:

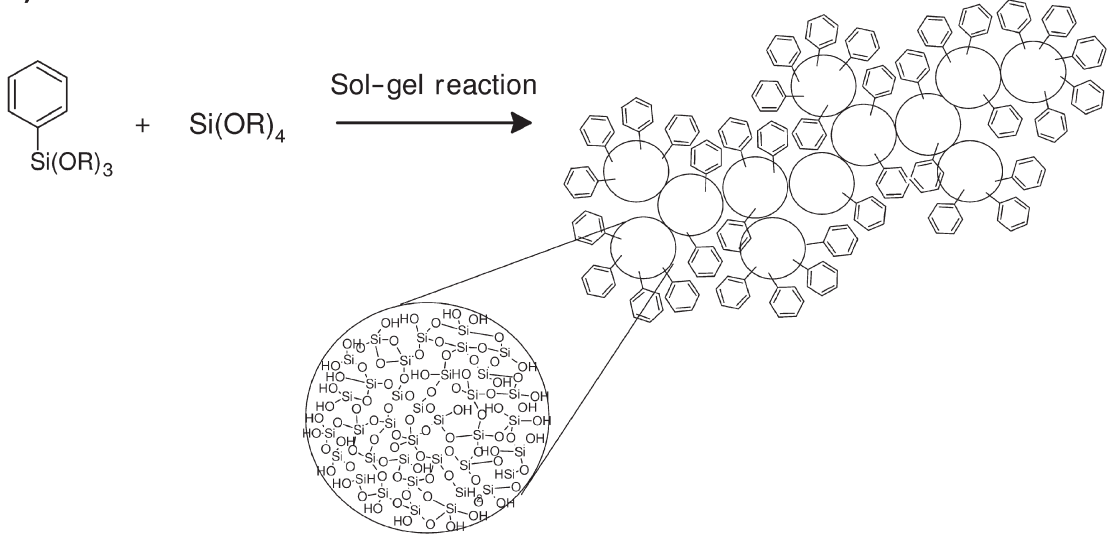

b) Network Builder:
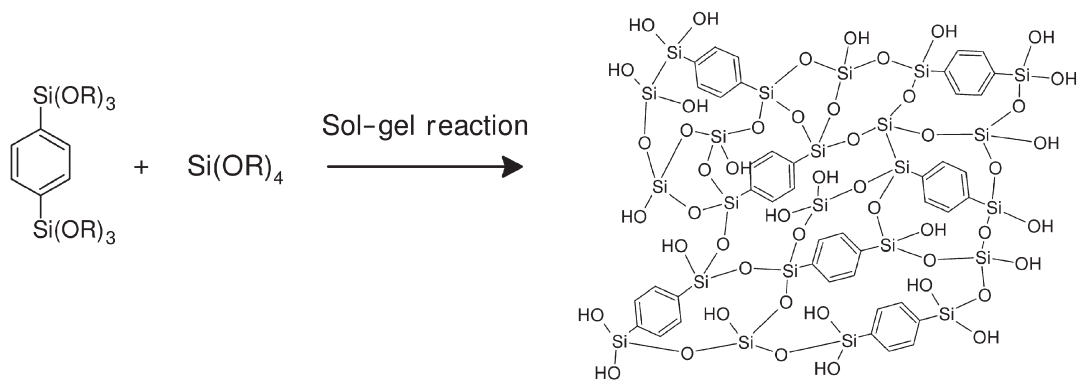

c) Network Functionalizer:

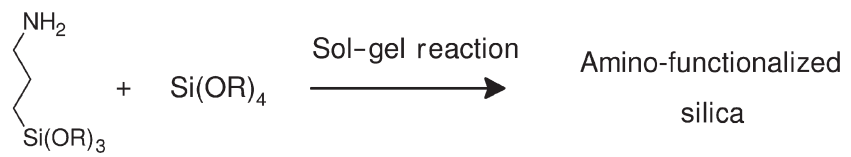

Scheme 1.1 Role of organically functionalized trialkoxysilanes in the silicon-based sol-gel process.

organic polymers (Scheme 1.2c) or inorganic and organic polymers are covalently connected with each other (Scheme 1.2d).

Nanocomposites After having discussed the above examples one question arises: what is the difference between inorganic-organic hybrid materials and inorganic-organic nanocomposites? In fact there is no clear borderline between these materials. The term nanocomposite is used if one of the structural units, either the organic or the inorganic, is in a defined size range of 1-100 nm. Therefore there is a gradual transition between hybrid materials and nanocomposites, 
Class I Hybrids

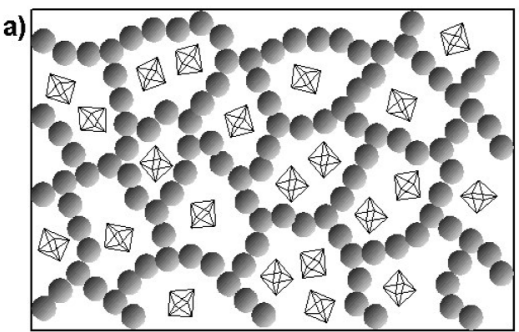

Blends

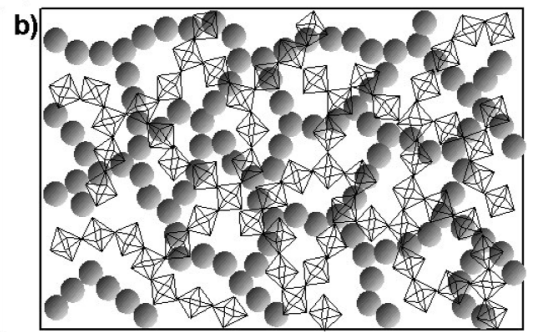

Interpenetrating networks

Class II Hybrids

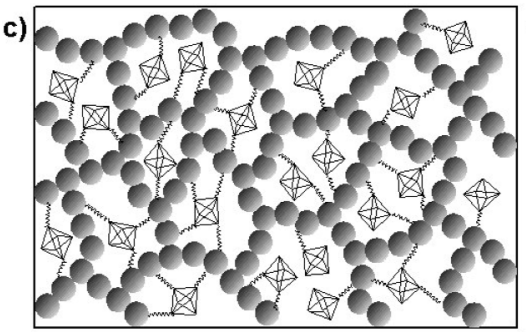

Building blocks covalently connected

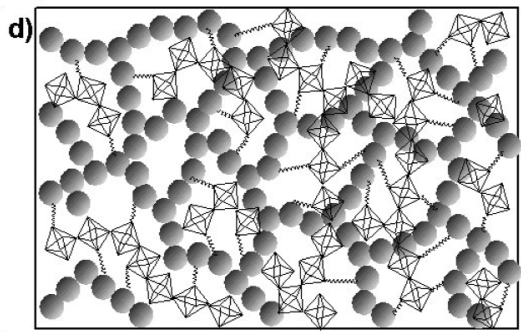

Covalently connected polymers

Scheme 1.2 The different types of hybrid materials.

because large molecular building blocks for hybrid materials, such as large inorganic clusters, can already be of the nanometer length scale. Commonly the term nanocomposites is used if discrete structural units in the respective size regime are used and the term hybrid materials is more often used if the inorganic units are formed in situ by molecular precursors, for example applying sol-gel reactions. Examples of discrete inorganic units for nanocomposites are nanoparticles, nanorods, carbon nanotubes and galleries of clay minerals (Fig. 1.2). Usually a nanocomposite is formed from these building blocks by their incorporation in organic polymers. Nanocomposites of nanoparticles are discussed in more detail in Chapter 2 and those incorporating clay minerals in Chapter 4.

\section{1 .4}

\section{Advantages of Combining Inorganic and Organic Species in One Material}

The most obvious advantage of inorganic-organic hybrids is that they can favorably combine the often dissimilar properties of organic and inorganic components in one material (Table 1.3). Because of the many possible combinations of components this field is very creative, since it provides the opportunity to invent an almost unlimited set of new materials with a large spectrum of known and as yet unknown properties. Another driving force in the area of hybrid materials is the possibility to create multifunctional materials. Examples are the incorporation of 
a)

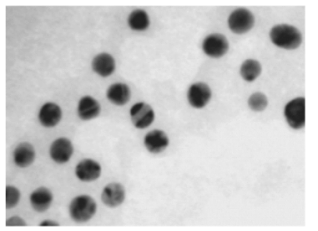

b)

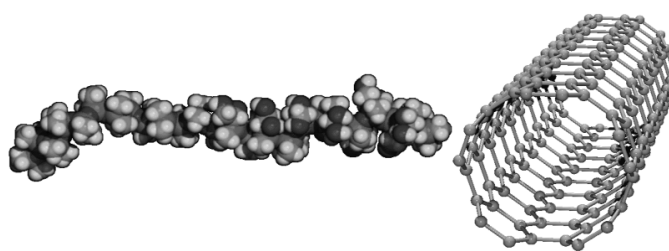

d)

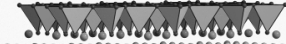

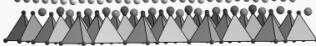

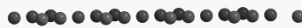

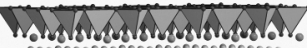

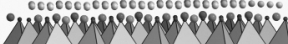

Fig. 1.2 Inorganic building blocks used for embedment in an organic matrix in the preparation of inorganic-organic nanocomposites: a) nanoparticles, b) macromolecules, c) nanotubes, d) layered materials.

Table 1.3 Comparison of general properties of typical inorganic and organic materials.

\begin{tabular}{|c|c|c|}
\hline Properties & Organics (polymers) & $\begin{array}{l}\text { Inorganics }\left(\mathrm{SiO}_{2}, \text { transition }\right. \\
\text { metal oxides (TMO)) }\end{array}$ \\
\hline Nature of bonds & $\begin{array}{l}\text { covalent }[\mathrm{C}-\mathrm{C}] \text {, van der Waals, } \\
\text { H-bonding }\end{array}$ & ionic or iono-covalent $[\mathrm{M}-\mathrm{O}]$ \\
\hline$T_{\mathrm{g}}$ & low $\left(-120^{\circ} \mathrm{C}\right.$ to $\left.200^{\circ} \mathrm{C}\right)$ & high $\left(\gg 200^{\circ} \mathrm{C}\right)$ \\
\hline Thermal stability & low $\left(<350^{\circ} \mathrm{C}-450^{\circ} \mathrm{C}\right)$ & high $\left(\gg 100^{\circ} \mathrm{C}\right)$ \\
\hline Density & $0.9-1.2$ & $2.0-4.0$ \\
\hline Refractive index & $1.2-1.6$ & $1.15-2.7$ \\
\hline Mechanical properties & $\begin{array}{l}\text { elasticity } \\
\text { plasticity } \\
\text { rubbery (depending on } T_{\mathrm{g}} \text { ) }\end{array}$ & $\begin{array}{l}\text { hardness } \\
\text { strength } \\
\text { fragility }\end{array}$ \\
\hline Hydrophobicity & hydrophilic & hydrophilic \\
\hline Permeability & $\begin{array}{l}\text { hydrophobic } \\
\pm \text { permeable to gases }\end{array}$ & low permeability to gases \\
\hline Electronic properties & $\begin{array}{l}\text { insulating to conductive } \\
\text { redox properties }\end{array}$ & $\begin{array}{l}\text { insulating to semiconductors } \\
\left(\mathrm{SiO}_{2}, \mathrm{TMO}\right) \\
\text { redox properties (TMO) } \\
\text { magnetic properties }\end{array}$ \\
\hline Processability & $\begin{array}{l}\text { high (molding, casting, film } \\
\text { formation, control of viscosity) }\end{array}$ & $\begin{array}{l}\text { low for powders } \\
\text { high for sol-gel coatings }\end{array}$ \\
\hline
\end{tabular}


inorganic clusters or nanoparticles with specific optical, electronic or magnetic properties in organic polymer matrices. These possibilities clearly reveal the power of hybrid materials to generate complex systems from simpler building blocks in a kind of LEGO (C) approach.

Probably the most intriguing property of hybrid materials that makes this material class interesting for many applications is their processing. Contrary to pure solid state inorganic materials that often require a high temperature treatment for their processing, hybrid materials show a more polymer-like handling, either because of their large organic content or because of the formation of crosslinked inorganic networks from small molecular precursors just like in polymerization reactions. Hence, these materials can be shaped in any form in bulk and in films. Although from an economical point of view bulk hybrid materials can currently only compete in very special areas with classical inorganic or organic materials, e.g. in the biomaterials sector, the possibility of their processing as thin films can lead to property improvements of cheaper materials by a simple surface treatment, e.g. scratch resistant coatings.

Based on the molecular or nanoscale dimensions of the building blocks, light scattering in homogeneous hybrid material can be avoided and therefore the optical transparency of the resulting hybrid materials and nanocomposites is, dependent on the composition used, relatively high. This makes these materials ideal candidates for many optical applications (Chapter 9). Furthermore, the materials' building blocks can also deliver an internal structure to the material which can be regularly ordered. While in most cases phase separation is avoided, phase separation of organic and inorganic components is used for the formation of porous materials, as described in Chapter 5.

Material properties of hybrid materials are usually changed by modifications of the composition on the molecular scale. If, for example, more hydrophobicity of a material is desired, the amount of hydrophobic molecular components is increased. In sol-gel materials this is usually achieved if alkyl- or aryl-substituted trialkoxysilanes are introduced in the formulation. Hydrophobic and lipophobic materials are composed if partially or fully fluorinated molecules are included. Mechanical properties, such as toughness or scratch resistance, are tailored if hard inorganic nanoparticles are included into the polymer matrix. Because the compositional variations are carried out on the molecular scale a gradual fine tuning of the material properties is possible.

One important subject in materials chemistry is the formation of smart materials, such as materials that react to environmental changes or switchable systems, because they open routes to novel technologies, for example electroactive materials, electrochromic materials, sensors and membranes, biohybrid materials, etc. The desired function can be delivered from the organic or inorganic or from both components. One of the advantages of hybrid materials in this context is that functional organic molecules as well as biomolecules often show better stability and performance if introduced in an inorganic matrix. 


\section{1 .5 \\ Interface-determined Materials}

The transition from the macroscopic world to microscopic, nanoscopic and molecular objects leads, beside the change of physical properties of the material itself, i.e. the so called quantum size effects, to the change of the surface area of the objects. While in macroscopic materials the majority of the atoms is hidden in the bulk of the material it becomes vice versa in very small objects. This is demonstrated by a simple mind game (Fig. 1.3). If one thinks of a cube of atoms in tight packing of $16 \times 16 \times 16$ atoms. This cube contains an overall number of 4096 atoms from which 1352 are located on the surface ( $33 \%$ surface atoms); if this cube is divided into eight equal $8 \times 8 \times 8$ cubes the overall number is the same but 2368 atoms are now located on the surface ( $58 \%$ surface atoms); repeating this procedure we get 3584 surface atoms ( $88 \%$ surface atoms). This example shows how important the surface becomes when objects become very small. In small nanoparticles $(<10 \mathrm{~nm})$ nearly every atom is a surface atom that can interact with the environment. One predominant feature of hybrid materials or nanocomposites is their inner interface, which has a direct impact on the properties of the different building blocks and therefore on the materials' properties. As already explained in Section 1.1.3, the nature of the interface has been used to divide the materials in two classes dependent on the strength of interaction between the moieties. If the two phases have opposite properties, such as different polarity, the system would thermodynamically phase separate. The same can happen on the molecular or nanometer level, leading to microphase separation. Usually, such a system would thermodynamically equilibrate over time. However in many cases in hybrid materials the system is kinetically stabilized by networkforming reactions such as the sol-gel process leading to a spatial fixation of the structure. The materials formed can be macroscopically homogeneous and optically clear, because the phase segregation is of small length scale and therefore limited interaction with visible light occurs. However, the composition on the molecular or nanometer length scale can be heterogeneous. If the phase segregation
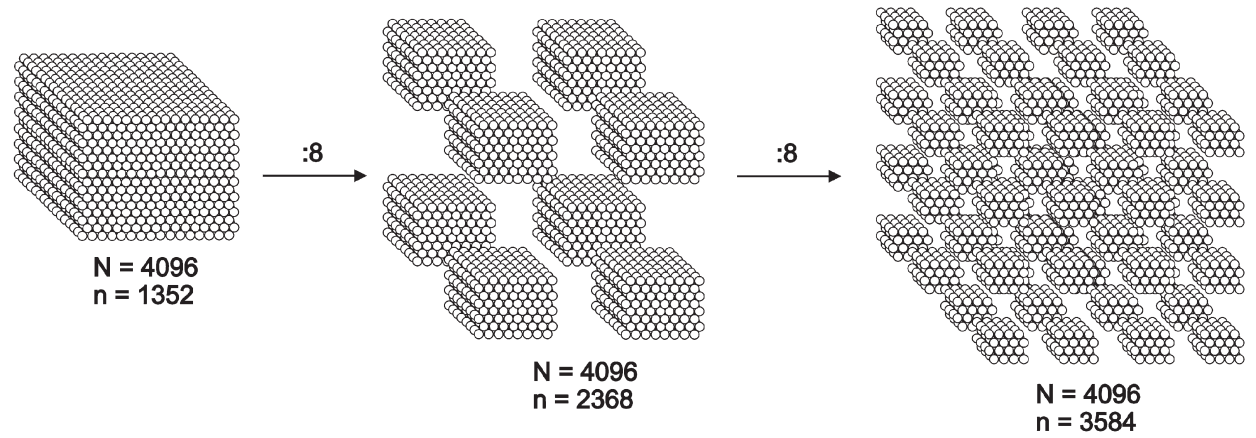

Fig. 1.3 Surface statistical consequences of dividing a cube with $16 \times 16 \times 16$ atoms. $\mathrm{N}=$ total atoms, $\mathrm{n}=$ surface atoms. 
reaches the several hundred nanometer length scale or the refractive index of the formed domains is very different, materials often turn opaque. Effects like this are avoided if the reaction parameters are controlled in such a way that the speed of network formation is kept faster than the phase separation reactions.

The high surface area of nanobuilding blocks can lead to additional effects; for example if surface atoms strongly interact with molecules of the matrix by chemical bonding, reactions like surface reorganization, electron transfer, etc. can occur which can have a large influence on the physical properties of the nano-building blocks and thus the overall performance of the material formed. It is, for example, known that conjugated $\pi$-electron systems coordinated to the surface of titania nanoparticles can lead to charge transfer reactions that influence the color, and therefore the surface electronic properties of the particles.

Nanosized objects, such as inorganic nanoparticles, in addition show a very high surface energy. Usually if the surface energy is not reduced by surface active agents (e.g. surfactants), such particles tend to agglomerate in an organic medium. Thus, the physical properties of the nanoparticles (e.g. quantum size effects) diminish, and/or the resulting materials are no longer homogeneous. Both facts have effects on the final material properties. For example the desired optical properties of nanocomposites fade away, or mechanical properties are weakened. However, sometimes a controlled aggregation can also be required, e.g. percolation of conducting particles in a polymer matrix increases the overall conductivity of the material (see Chapter 10).

\section{1 .6}

\section{The Role of the Interaction Mechanisms}

In Section 1.1.3 the interaction mechanism between the organic and inorganic species was used to categorize the different types of hybrid materials, furthermore of course the interaction also has an impact on the material properties. Weak chemical interactions between the inorganic and organic entities leave some potential for dynamic phenomena in the final materials, meaning that over longer periods of time changes in the material, such as aggregation, phase separation or leaching of one of the components, can occur. These phenomena can be avoided if strong interactions are employed such as covalent bonds, as in nanoparticlecrosslinked polymers. Depending on the desired materials' properties the interactions can be gradually tuned. Weak interactions are, for example, preferred where a mobility of one component in the other is required for the target properties. This is for example the case for ion conducting polymers, where the inorganic ion (often $\mathrm{Li}^{+}$) has to migrate through the polymer matrix.

In many examples the interactions between the inorganic and organic species are maximized by applying covalent attachment of one to the other species. But there are also cases where small changes in the composition, which on the first sight should not result in large effects, can make considerable differences. It was, for example, shown that interpenetrating networks between polystyrene and sol-gel materials modified with phenyl groups show less microphase segregation 
than sol-gel materials with pure alkyl groups, which was interpreted to be an effect of $\pi$ - $\pi$-interactions between the two materials.

In addition the interaction of the two components can have an influence on other properties, such as electronic properties if coordination complexes are formed or electron transfer processes are enabled by the interaction.

\section{2}

\section{Synthetic Strategies towards Hybrid Materials}

In principle two different approaches can be used for the formation of hybrid materials: Either well-defined preformed building blocks are applied that react with each other to form the final hybrid material in which the precursors still at least partially keep their original integrity or one or both structural units are formed from the precursors that are transformed into a novel (network) structure.

Both methodologies have their advantages and disadvantages and will be described here in more detail.

Building block approach As mentioned above building blocks at least partially keep their molecular integrity throughout the material formation, which means that structural units that are present in these sources for materials formation can also be found in the final material. At the same time typical properties of these building blocks usually survive the matrix formation, which is not the case if material precursors are transferred into novel materials. Representative examples of such well-defined building blocks are modified inorganic clusters or nanoparticles with attached reactive organic groups (Fig. 1.4).

Cluster compounds often consist of at least one functional group that allows an interaction with an organic matrix, for example by copolymerization. Depending on the number of groups that can interact, these building blocks are able to modify an organic matrix (one functional group) or form partially or fully crosslinked materials (more than one group). For instance, two reactive groups can lead to the formation of chain structures. If the building blocks contain at least three reactive groups they can be used without additional molecules for the formation of a crosslinked material.

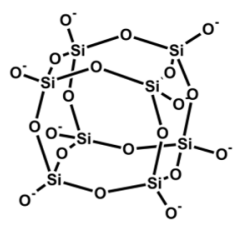

Spherosilicates

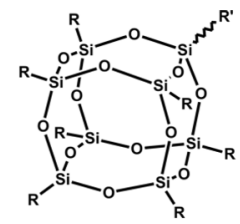

Polyhedral oligomeric silsesquioxanes (POSS)

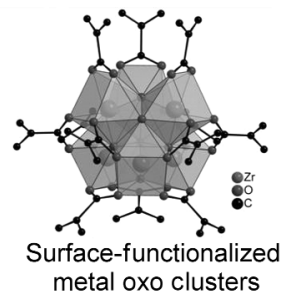

metal oxo clusters

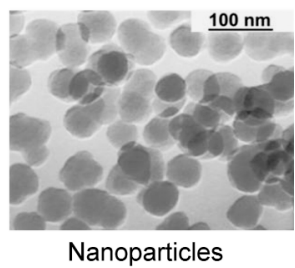

Nanoparticles

Fig. 1.4 Typical well-defined molecular building blocks used in the formation of hybrid materials. 
Beside the molecular building blocks mentioned, nanosized building blocks, such as particles or nanorods, can also be used to form nanocomposites. The building block approach has one large advantage compared with the in situ formation of the inorganic or organic entities: because at least one structural unit (the building block) is well-defined and usually does not undergo significant structural changes during the matrix formation, better structure-property predictions are possible. Furthermore, the building blocks can be designed in such a way to give the best performance in the materials' formation, for example good solubility of inorganic compounds in organic monomers by surface groups showing a similar polarity as the monomers.

In situ formation of the components Contrary to the building block approach the in situ formation of the hybrid materials is based on the chemical transformation of the precursors used throughout materials' preparation. Typically this is the case if organic polymers are formed but also if the sol-gel process is applied to produce the inorganic component. In these cases well-defined discrete molecules are transformed to multidimensional structures, which often show totally different properties from the original precursors. Generally simple, commercially available molecules are applied and the internal structure of the final material is determined by the composition of these precursors but also by the reaction conditions. Therefore control over the latter is a crucial step in this process. Changing one parameter can often lead to two very different materials. If, for example, the inorganic species is a silica derivative formed by the sol-gel process, the change from base to acid catalysis makes a large difference because base catalysis leads to a more particle-like microstructure while acid catalysis leads to a polymer-like microstructure. Hence, the final performance of the derived materials is strongly dependent on their processing and its optimization.

\subsection{1}

\section{In situ Formation of Inorganic Materials}

Many of the classical inorganic solid state materials are formed using solid precursors and high temperature processes, which are often not compatible with the presence of organic groups because they are decomposed at elevated temperatures. Hence, these high temperature processes are not suitable for the in situ formation of hybrid materials. Reactions that are employed should have more the character of classical covalent bond formation in solutions. One of the most prominent processes which fulfill these demands is the sol-gel process. However, such rather low temperature processes often do not lead to the thermodynamically most stable structure but to kinetic products, which has some implications for the structures obtained. For example low temperature derived inorganic materials are often amorphous or crystallinity is only observed on a very small length scale, i.e. the nanometer range. An example of the latter is the formation of metal nanoparticles in organic or inorganic matrices by reduction of metal salts or organometallic precursors. 


\subsubsection{Sol-Gel Process}

This process is chemically related to an organic polycondensation reaction in which small molecules form polymeric structures by the loss of substituents. Usually the reaction results in a three-dimensional (3-D) crosslinked network. The fact that small molecules are used as precursors for the formation of the crosslinked materials implies several advantages, for example a high control of the purity and composition of the final materials and the use of a solvent based chemistry which offers many advantages for the processing of the materials formed.

The silicon-based sol-gel process is probably the one that has been most investigated; therefore the fundamental reaction principles are discussed using this process as a model system. One important fact also makes the silicon-based sol-gel processes a predominant process in the formation of hybrid materials, which is the simple incorporation of organic groups using organically modified silanes. $\mathrm{Si}-\mathrm{C}$ bonds have enhanced stability against hydrolysis in the aqueous media usually used, which is not the case for many metal-carbon bonds, so it is possible to easily incorporate a large variety of organic groups in the network formed. Principally $\mathrm{R}_{4-\mathrm{n}} \mathrm{SiX}_{\mathrm{n}}$ compounds ( $\mathrm{n}=1-4, \mathrm{X}=\mathrm{OR}$ ', halogen) are used as molecular precursors, in which the $\mathrm{Si}-\mathrm{X}$ bond is labile towards hydrolysis reactions forming unstable silanols $(\mathrm{Si}-\mathrm{OH})$ that condensate leading to $\mathrm{Si}-\mathrm{O}-\mathrm{Si}$ bonds. In the first steps of this reaction oligo- and polymers as well as cyclics are formed subsequently resulting in colloids that define the sol. Solid particles in the sol afterwards undergo crosslinking reactions and form the gel (Scheme 1.3).
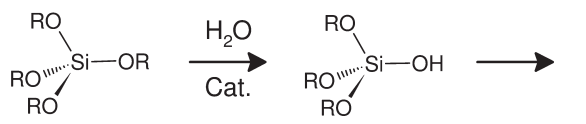<smiles>[2H][PbH]([O-])O[Si](O)(O)O</smiles><smiles>CC[18O][R]([18OH])([18OH])O[Si](O)(O)O</smiles>
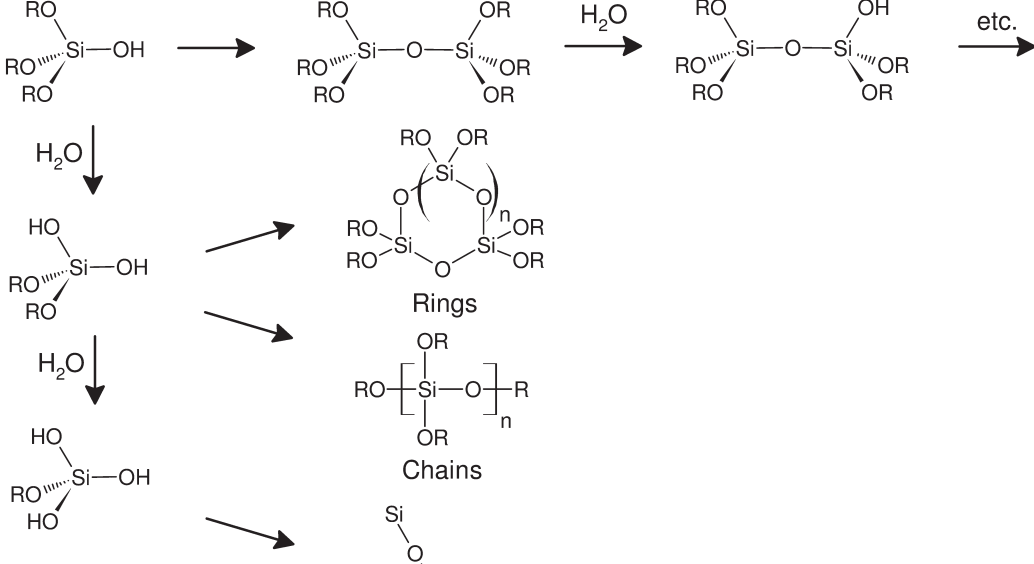

Chains

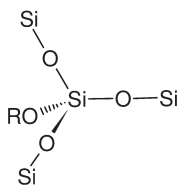

Scheme 1.3 Fundamental reaction steps in the sol-gel process based on tetrialkoxysilanes.

The process is catalyzed by acids or bases resulting in different reaction mechanisms by the velocity of the condensation reaction (Scheme 1.4). The $\mathrm{pH}$ used therefore has an effect on the kinetics which is usually expressed by the gel point 
Acid Catalysis:

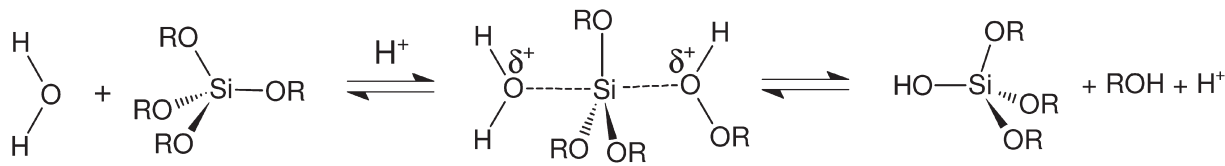

Base Catalysis:

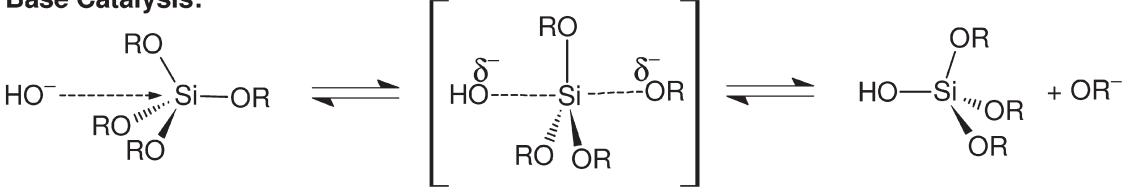

Scheme 1.4 Differences in mechanism depending on the type of catalyst used in the silicon-based sol-gel process.

of the sol-gel reaction. The reaction is slowest at the isoelectric point of silica (between 2.5 and 4.5 depending on different parameters) and the speed increases rapidly on changing the $\mathrm{pH}$. Not only do the reaction conditions have a strong influence on the kinetics of the reaction but also the structure of the precursors. Generally, larger substituents decrease the reaction time due to steric hindrance. In addition, the substituents play a mature role in the solubility of the precursor in the solvent. Water is required for the reaction and if the organic substituents are quite large usually the precursor becomes immiscible in the solvent. By changing the solvent one has to take into account that it can interfere in the hydrolysis reaction, for example alcohols can undergo trans-esterification reactions leading to quite complicated equilibria in the mixture. Hence, for a well-defined material the reaction conditions have to be fine-tuned.

The $\mathrm{pH}$ not only plays a major role in the mechanism but also for the microstructure of the final material. Applying acid-catalyzed reactions an open network structure is formed in the first steps of the reaction leading to condensation of small clusters afterwards. Contrarily, the base-catalyzed reaction leads to highly crosslinked sol particles already in the first steps. This can lead to variations in the homogeneity of the final hybrid materials as will be shown later. Commonly used catalysts are $\mathrm{HCl}, \mathrm{NaOH}$ or $\mathrm{NH}_{4} \mathrm{OH}$, but fluorides can be also used as catalysts leading to fast reaction times.

The transition from a sol to a gel is defined as the gelation point, which is the point when links between the sol particles are formed to such an extent that a solid material is obtained containing internal pores that incorporate the released alcohol. However at this point the reaction has not finished, but condensation reactions can go on for a long time until a final stage is reached. This process is called ageing. During this reaction the material shrinks and stiffens. This process is carried on in the drying process, where the material acquires a more compact structure and the associated crosslinking leads to an increased stiffness. During the drying process the large capillary forces of the evaporating liquids in the porous structure take place which can lead to cracking of the materials. Reaction parameters such as drying rate, gelation time, $\mathrm{pH}$, etc. can have a major influence on 
the cracking of the gels and have therefore to be optimized. Under some circumstances the destruction of the gel network can lead to the formation of powders instead of monoliths during materials formation.

Stress during the drying process can be avoided if the liquid in the pores is exchanged under supercritical conditions where the distinction between liquid and vapor no longer exists. This process leads to so-called highly porous aerogels compared with the conventionally dried xerogels.

As already mentioned above many parameters influence the speed of the sol-gel reaction; if a homogeneous material is required all parameters must be optimized. This is particularly true if hybrid materials are the target, because undesired phase separations of organic and inorganic species in the materials or between the network and unreacted precursors weaken the materials' properties. This can often even be observed by the naked eye if the material turns opaque.

The water to precursor ratio is also a major parameter in the sol-gel process. If tetraalkoxysilanes are used as precursors, two water molecules per starting compound are necessary to form completely condensed $\mathrm{SiO}_{2}$. Applying a lower $\mathrm{H}_{2} \mathrm{O} / \mathrm{Si}$ ratio, would lead to an alkoxide containing final material.

\subsubsection{Nonhydrolytic Sol-Gel Process}

In this process the reaction between metal halides and alkoxides is used for the formation of the products (Scheme 1.5). The alkoxides can be formed during the process by various reactions. Usually this process is carried out in sealed tubes at elevated temperature but it can also be employed in unsealed systems under an inert gas atmosphere.

\section{Non-hydrolytic sol-gel process:}

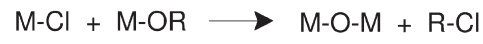

In situ formation of alkoxides:

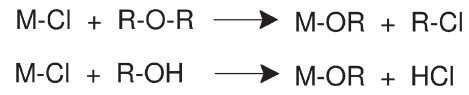

Scheme 1.5 Mechanisms involved in the nonhydrolytic sol-gel process.

\subsubsection{Sol-Gel Reactions of Non-Silicates}

Metal and transition-metal alkoxides are generally more reactive towards hydrolysis and condensation reactions compared with silicon. The metals in the alkoxides are usually in their highest oxidation state surrounded by electronegative -OR ligands which render them susceptible to nucleophilic attack. Transition metal alkoxides show a lower electronegativity compared with silicon which causes them to be more electrophilic and therefore less stable towards hydrolysis in the sol-gel reactions. Furthermore, transition metals often show several stable coordination environments. While the negatively charged alkoxides balance the charge of the metal cation they generally cannot completely saturate the coordination sphere of 
<smiles></smiles>

Carboxylates

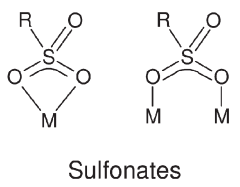<smiles>[R]C1=NOCC([R])=C1[R]</smiles>

$\beta$-Diketonates

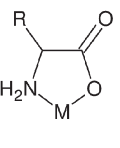

$\alpha$-Aminocarboxylates

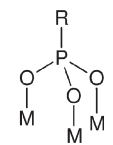

Phosphonates

Fig. 1.5 Typical coordination patterns between bi- and multidentate ligands and metals that can be applied for the incorporation of organic functionalities in metal oxides.

the metals, which leads to the formation of oligomers via alkoxide or alcohol bridges and/or the saturation of the coordination environment by additional coordination of alcohol molecules, which also has an impact on the reactivity of the metal alkoxides. More sterically demanding alkoxides, such as isopropoxides, lead to a lower degree of aggregation and smaller alkoxides, such as ethoxides or $n$-propoxides, to a larger degree of aggregation. In addition, the length of the alkyl group in the metal alkoxides also influences their solubility in organic solvents, for example ethoxides often show a much lower solubility as their longer alkyl chain containing homologs.

As already mentioned $\mathrm{M}-\mathrm{C}$ bonds in metal alkoxides are in most cases not stable enough to survive the sol-gel conditions. Therefore, contrary to the silica route, other mechanisms have to be employed if it is desired that hybrid inorganic-organic metal oxide materials be formed in a one-step approach. One solution to the latter problem is the use of organically functionalized bi- and multidentate ligands that show a higher bonding stability during the sol-gel reaction and, in addition, reduce the speed of the reaction by blocking coordination sites (Fig. 1.5).

\subsubsection{Hybrid Materials by the Sol-Gel Process}

Organic molecules other than the solvent can be added to the sol and become physically entrapped in the cavities of the formed network upon gelation. For this purpose the molecules have to endure the reaction conditions of the sol-gel process, namely the aqueous conditions and the $\mathrm{pH}$ of the environment. Hence, functional organic groups that can be hydrolyzed are not tolerated, but a partial tolerance for the $\mathrm{pH}$ can be obtained if the sol-gel reaction is carried out in a buffer solution. This is particularly necessary if biological molecules, such as enzymes, are to be entrapped in the gel. Physical entrapment has the disadvantage that sometimes the materials obtained are not stable towards phase separation or leaching because of differences in polarity. Chemical modification of organic compounds with trialkoxysilane groups can partially avoid such problems due to co-condensation during the formation of the sol-gel network and thus development of covalent linkages to the network. Trialkoxysilane groups are typically introduced by a platinum catalyzed reaction between an unsaturated bond and a trialkoxysilane (Scheme 1.6). 


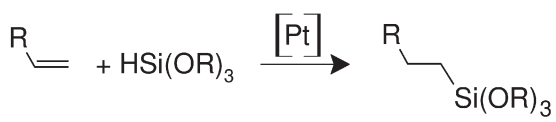

Scheme 1.6 Platinum catalyzed hydrosilation for the introduction of trialkoxysilane groups.

While the formation of homogeneous materials with a chemical link between the inorganic and organic component is in many cases the preferred route, there are cases where a controlled phase separation between the entrapped organic molecules and the sol-gel material is compulsory for the formation of the material, for example in the preparation of mesoporous materials (Chapter 5).

Besides the entrapment of organic systems, precursors with hydrolytically stable $\mathrm{Si}-\mathrm{C}$ bonds can also be used for co-condensation reactions with tetraalkoxysilanes. In addition, organically functionalized trialkoxysilanes can also be used for the formation of 3-D networks alone forming so called silsesquioxanes (general formula $\mathrm{R}-\mathrm{SiO}_{1.5}$ ) materials. Generally a 3-D network can only be obtained if three or more hydrolyzable bonds are present in a molecule. Two such bonds generally result in linear products and one bond leads only to dimers or allows a modification of a preformed network by the attachment to reactive groups on the surface of the inorganic network (Fig. 1.6). Depending on the reaction conditions in the sol-gel process smaller species are also formed in the organotrialkoxysilanebased sol-gel process, for example cage structures or ladder-like polymers (Fig. 1.6). Because of the stable $\mathrm{Si}-\mathrm{C}$ bond the organic unit can be included within the silica matrix without transformation. There are only a few $\mathrm{Si}-\mathrm{C}$ bonds that are not stable against hydrolysis, for example the $\mathrm{Si}-\mathrm{C} \equiv \mathrm{C}$ bond where the $\mathrm{Si}-$ $\mathrm{C}$ bond can be cleaved by $\mathrm{H}_{2} \mathrm{O}$ if fluoride ions are present. Some typical examples for trialkoxysilane compounds used in the formation of hybrid materials are shown in Scheme 1.7. Usually the organic functionalizations have a large influence on the properties of the final hybrid material. First of all the degree of condensation of a hybrid material prepared by trialkoxysilanes is generally smaller than in the case of tetraalkoxysilanes and thus the network density is also reduced.<smiles></smiles><smiles>[2H][Si]([2H])([2H])[O-]</smiles><smiles>[2H][Si]([2H])([18OH])P</smiles>

$\mathrm{SiO}_{2}$

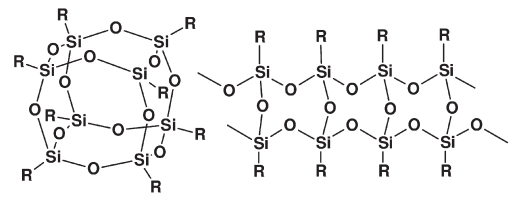

Silica

Silsesquioxanes

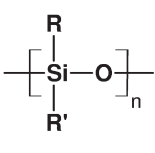

Oligo- and polysiloxanes

Fig. 1.6 Formation of different structures during hydrolysis in dependence of the number of organic substituents compared to labile substituents at the silicon atom. 
In addition, the functional group incorporated changes the properties of the final material, for example fluoro-substituted compounds can create hydrophobic and lipophobic materials, additional reactive functional groups can be introduced to allow further reactions such as amino, epoxy or vinyl groups (Scheme 1.7). Beside molecules with a single trialkoxysilane group also multifunctional organic molecules can be used, which are discussed in more detail in Chapter 6.
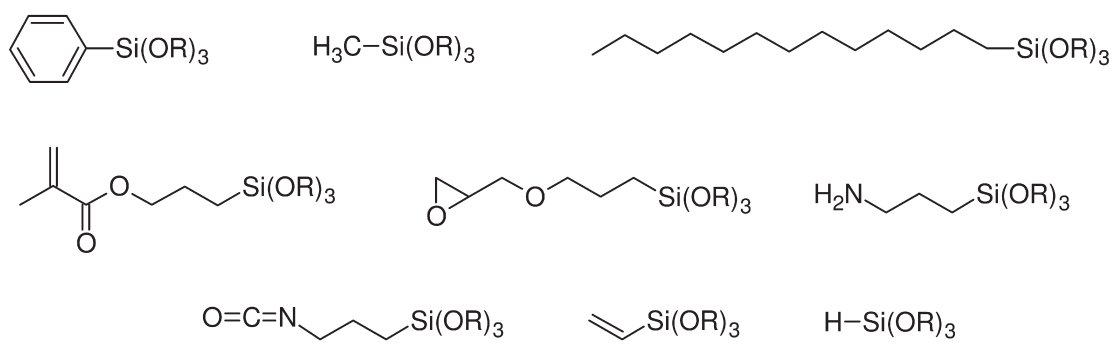

Scheme 1.7 Trialkoxysilane precursors often used in the sol-gel process.

More detailed discussions of the sol-gel process can be found in the cited literature.

\subsubsection{Hybrid Materials Derived by Combining the Sol-Gel Approach and Organic Polymers}

Compared with other inorganic network forming reactions, the sol-gel processes show mild reaction conditions and a broad solvent compatibility. These two characteristics offer the possibility to carry out the inorganic network forming process in presence of a preformed organic polymer or to carry out the organic polymerization before, during or after the sol-gel process. The properties of the final hybrid materials are not only determined by the properties of the inorganic and organic component, but also by the phase morphology and the interfacial region between the two components. The often dissimilar reaction mechanisms of the sol-gel process and typical organic polymerizations, such as free radical polymerizations, allow the temporal separation of the two polymerization reactions which offers many advantages in the material formation.

One major parameter in the synthesis of these materials is the identification of a solvent in which the organic macromolecules are soluble and which is compatible with either the monomers or preformed inorganic oligomers derived by the sol-gel approach. Many commonly applied organic polymers, such as polystyrene or polymethacrylates, are immiscible with alcohols that are released during the sol-gel process and which are also used as solvents, therefore phase separation is enforced in these cases. This can be avoided if the solvent is switched from the typically used alcohols to, for example, THF in which many organic polymers are soluble and which is compatible with many sol-gel reactions. Phase separation can also be avoided if the polymers contain functional groups that are more compatible with the reaction conditions of the sol-gel process or even undergo an interaction with the inorganic material formed. This can be achieved, for example 
by the incorporation of $\mathrm{OH}$-groups that interact with, for example, hydroxyl groups formed during the sol-gel process or by ionic modifications of the organic polymer. Covalent linkages can be formed if functional groups that undergo hydrolysis and condensation reactions are covalently attached to the organic monomers. Some typically used monomers that are applied in homo- or copolymerizations are shown in Scheme 1.8.<smiles>C=C(C)C(=O)OCCO</smiles><smiles>C=Cc1ccncc1</smiles><smiles>C=CC(=O)N(C)C</smiles>

Hydroxyethyl methacrylate (HEMA)

Vinylpyridine Dimethylacetamide<smiles>OCCCOCC1CO1</smiles><smiles>C=C(C)C(=O)OCCCO</smiles>

\section{Glycidoxypropyl-} trialkoxysilane

\section{Methacroylpropyl -trialkoxysilane}

Scheme 1.8 Organic monomers typically applied in the formation of sol-gel/organic polymer hybrid materials.

\section{2 .2}

\section{Formation of Organic Polymers in Presence of Preformed Inorganic Materials}

If the organic polymerization occurs in the presence of an inorganic material to form the hybrid material one has to distinguish between several possibilities to overcome the incompatibilty of the two species. The inorganic material can either have no surface functionalization but the bare material surface; it can be modified with nonreactive organic groups (e.g. alkyl chains); or it can contain reactive surface groups such as polymerizable functionalities. Depending on these prerequisites the material can be pretreated, for example a pure inorganic surface can be treated with surfactants or silane coupling agents to make it compatible with the organic monomers, or functional monomers can be added that react with the surface of the inorganic material. If the inorganic component has nonreactive organic groups attached to its surface and it can be dissolved in a monomer which is subsequently polymerized, the resulting material after the organic polymerization, is a blend. In this case the inorganic component interact only weakly or not at all with the organic polymer; hence, a class I material is formed. Homogeneous materials are only obtained in this case if agglomeration of the inorganic compo- 
nents in the organic environment is prevented. This can be achieved if the interactions between the inorganic components and the monomers are better or at least the same as between the inorganic components. However, if no strong chemical interactions are formed, the long-term stability of a once homogeneous material is questionable because of diffusion effects in the resulting hybrid material. Examples of such materials are alkyl chain functionalized silica nanoparticles that can be introduced into many hydrophobic polymers, the use of block copolymers containing a poly(vinyl pyridine) segment that can attach to many metal nanoparticles, or the use of hydroxyethyl methacrylates in the polymerization mixture together with metal oxide nanoparticles. In the latter example hydrogen bridges are formed between the polymer matrix and the particle surface. The stronger the respective interaction between the components, the more stable is the final material. The strongest interaction is achieved if class II materials are formed, for example with covalent interactions. Examples for such strong interactions are the use of surface-attached polymerizable groups that are copolymerized with organic monomers. Some examples of such systems are shown in the Chapters 2 and 3.

If a porous 3-D inorganic network is used as the inorganic component for the formation of the hybrid material a different approach has to be employed depending on the pore size, the surface functionalization of the pores and the stiffness of the inorganic framework. In many cases intercalation of organic components into the cavities is difficult because of diffusion limits. Several porous or layered inorganic materials have already been used to prepare hybrid materials and nanocomposites. Probably the most studied materials, class in this respect is that of two-dimensional (2-D) layered inorganic materials that can intercalate organic molecules and if polymerization between the layers occurs even exfoliate, producing nanocomposites. Contrary to intercalated systems the exfoliated hybrids only contain a small weight percentage of host layers with no structural order. The preparation of such materials is described in more detail in Chapter 4 but principally three methods for the formation of polymer-clay nanocomposites can be used:

1. Intercalation of monomers followed by in situ polymerization

2. Direct intercalation of polymer chains from solution

3. Polymer melt intercalation

The method applied depends on the inorganic component and on the polymerization technique used and will not be discussed in this introductory chapter.

Contrary to the layered materials, which are able to completely delaminate if the forces produced by the intercalated polymers overcome the attracting energy of the single layers, this is not possible in the case of the stable 3-D framework structures, such as zeolites, molecular sieves and M41S-materials. The composites obtained can be viewed as host-guest hybrid materials. There are two possible routes towards this kind of hybrid material; (a) direct threading of preformed polymer through the host channels (soluble and melting polymers) which is usually limited by the size, conformation, and diffusion behavior of the polymers and, 
(b) the in situ polymerization in the pores and channels of the hosts. The latter is the most widely used method for the synthesis of such systems. Of course, diffusion of the monomers in the pores is a function of the pore size, therefore the pores in zeolites with pore sizes of several hundred picometers are much more difficult to use in such reactions than mesoporous materials with pore diameters of several nanometers. Two methods proved to be very valuable for the filling of the porous structures with monomers: one is the soaking of the materials in liquid monomers and the other one is the filling of the pores in the gas phase. A better uptake of the monomers by the inorganic porous materials is achieved if the pores are pre-functionalized with organic groups increasing the absorption of monomers on the concave surface. In principle this technique is similar to the increase of monomer absorption on the surface of silica nanoparticles by the surface functionalization with silane coupling agents.

Beside of well-defined 3-D porous structures, sol-gel networks are also inherently porous materials. Uniform homogeneous materials can be obtained if the solvent of the sol-gel process is a monomer for a polymerization. This can be polymerized in a second step after the sol-gel process has occurred. It is much more difficult to functionalize a dry porous xerogel or aerogel because here a stiff inorganic network has already formed and has to be filled again with organic monomers. Principally the same methods as in the case of the ordered 3-D networks can be used for this purpose. Infiltration of preformed polymers into sol-gel networks is as difficult as in the case of the well-ordered porous systems because of the difficulties connected with the slow diffusion of organic polymer chains into the porous inorganic network.

\section{2 .3}

\section{Hybrid Materials by Simultaneous Formation of Both Components}

Simultaneous formation of the inorganic and organic polymers can result in the most homogeneous type of interpenetrating networks. Usually the precursors for the sol-gel process are mixed with monomers for the organic polymerization and both processes are carried out at the same time with or without solvent. Applying this method, three processes are competing with each other: (a) the kinetics of the hydrolysis and condensation forming the inorganic phase, (b) the kinetics of the polymerization of the organic phase, and (c) the thermodynamics of the phase separation between the two phases. Tailoring the kinetics of the two polymerizations in such a way that they occur simultaneously and rapidly enough, phase separation is avoided or minimized. Additional parameters such as attractive interactions between the two moieties, as described above can also be used to avoid phase separation.

One problem that also arises from the simultaneous formation of both networks is the sensitivity of many organic polymerization processes for sol-gel conditions or the composition of the materials formed. Ionic polymerizations, for example, often interact with the precursors or intermediates formed in the sol-gel process. Therefore, they are not usually applied in these reactions; instead free radical poly- 

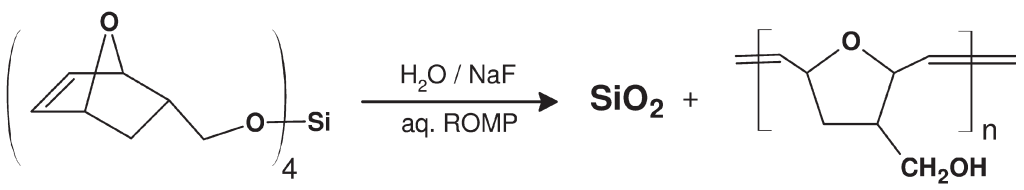<smiles>CC(C)=C(C)C(=O)OCCOC(C)(C)[Si]</smiles>

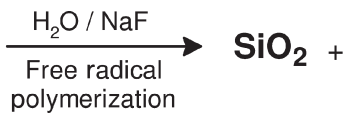<smiles>CC(C)(C)CC(C)(C(=O)OCCO)C(C)(C)C</smiles>

Fig. 1.7 Silicon sol-gel precursors with polymerizable alkoxides for ring opening metathesis polymerization (ROMP) or free radical polymerization.

merizations are the method of choice. This polymerization mechanism is very robust and can lead to very homogeneous materials. However, only selected, in particular vinyl, monomers can be used for this process. In addition, it is often also necessary to optimize the catalytic conditions of the sol-gel process. It is known, for example, that if the silicon sol-gel process is used basic catalysis leads to opaque final materials while the transparency can be improved if acidic conditions are used. This is most probably due to the different structures of the silica species obtained by the different approaches. While base catalysis leads to more particle-like networks that scatter light quite easily, acid catalysis leads to more polymer-like structures. Of course not only these parameters play a role for the transparency of the materials but also others such as the refractive index difference between organic polymer and inorganic species.

A very clever route towards hybrid materials by the sol-gel process is the use of precursors that contain alkoxides which also can act as monomers in the organic polymerization. The released alkoxides are incorporated in the polymers as the corresponding alcohol while the sol-gel process is carried out (Fig. 1.7). This leads to nanocomposites with reduced shrinkage and high homogeneity.

\subsection{4}

\section{Building Block Approach}

In recent years many building blocks have been synthesized and used for the preparation of hybrid materials. Chemists can design these compounds on a molecular scale with highly sophisticated methods and the resulting systems are used for the formation of functional hybrid materials. Many future applications, in particular in nanotechnology, focus on a bottom-up approach in which complex structures are hierarchically formed by these small building blocks. This idea is also one of the driving forces of the building block approach in hybrid materials. 
Another point which was also already mentioned is the predictability of the final material properties if well-defined building blocks are used.

A typical building block should consist of a well-defined molecular or nanosized structure and of a well-defined size and shape, with a tailored surface structure and composition. In regard of the preparation of functional hybrid materials the building block should also deliver interesting chemical or physical properties, in areas like conductivity, magnetic behavior, thermal properties, switching possibilities, etc. All these characteristics should be kept during the material formation, for example the embedment into a different phase. Building blocks can be inorganic or organic in nature, but because they are incorporated into another phase they should be somehow compatible with the second phase. Most of the times the compatibility is achieved by surface groups that allow some kind of interaction with a second component.

\subsubsection{Inorganic Building Blocks}

Prime examples of inorganic building blocks that can keep their molecular integrity are cluster compounds of various compositions. Usually clusters are defined as agglomerates of elements that either exclusively contain pure metals or metals in mixture with other elements. Although the classical chemical understanding of a cluster includes the existence of metal-metal bonds, the term cluster should be used in the context of this book in its meaning of an agglomerate of atoms in a given shape. Regularly pure metal clusters are not stable without surface functionalization with groups that decrease surface energy and thus avoid coalescence to larger particles. Both coalescence and surface reactivity of clusters are closely related to that of nanoparticles of the same composition. Because of this similarity and the fact that the transition from large clusters to small nanoparticles is fluent, we will not clearly distinguish between them. While in commonly applied metal clusters the main role of the coordinating ligands is the stabilization, they also can serve for a better compatibilization or interaction with an organic matrix. Similar mechanisms are valid for binary systems like metal chalcogenide or multicomponent clusters. Hence, the goal in the chemical design of these systems is the preparation of clusters carrying organic surface functionalizations that tailor the interface to an organic matrix by making the inorganic core compatible and by the addition of functional groups available for certain interactions with the matrix. One major advantage of the use of clusters is that they are small enough that usual chemical analysis methods such as liquid NMR spectroscopy and, if one is lucky, even single crystal X-ray diffraction can be used for their analysis. The high ratio between surface groups and volume makes it possible to get important information of the bonding situation in such systems and makes these compounds to essential models for larger, comparable systems, such as nanoparticles or surfaces.

Two methods are used for the synthesis of such surface-functionalized molecular building blocks: either the surface groups are grafted to a pre-formed cluster ("post-synthesis modification" method) or they are introduced during the cluster synthesis ("in-situ” method). 
Surface-functionalized metal clusters are one prominent model system for welldefined inorganic building blocks that can be used in the synthesis of hybrid materials. However, as with many other nanoscaled materials it is not possible to synthesize such pure clusters and to handle them without a specific surface coverage that limits the reactivity of the surface atoms towards agglomeration. From the aspect of the synthesis of hybrid materials this is no problem as long as the surface coverage of the cluster or nanoparticle contains the desired functionalities for an interaction with an organic matrix. A typical example of such a cluster is the phosphine-stabilized gold cluster of the type $\mathrm{Au}_{55}\left(\mathrm{PPh}_{3}\right)_{12} \mathrm{Cl}_{6}$ which is prepared by reduction of $\mathrm{AuCl}\left[\mathrm{PPh}_{3}\right]$ with diborane. Blocking of surface sites by $\mathrm{PPh}_{3}$ ligands guarantees the size limitation of these clusters and avoids their further growth. However, these phosphine-stabilized clusters are still unstable for example at elevated temperatures, which limits their applications. While triphenylphosphine only stabilizes the surface further functionalities can be included by an exchange of these capping agents. Ligand-exchange of the phosphine surface functionalization with alkyl- and arylthiols results in the corresponding thiolstabilized cluster. This exchange leads to a complete substitution of the $\mathrm{PPh}_{3}$ because of the better bonding capabilities of thiols to gold atoms compared with phosphines. A similar process is also used for the ligand exchange of stabilizing citrate groups on the surface of gold nanoparticles obtained by the reduction of $\mathrm{HAuCl}_{4}$ by sodium citrate. The mild conditions during the exchange process (simple stirring in an organic solvent at room temperature), allows the functionalization of the clusters with different functionalized thiol ligands. Similar surface functionalizations can be carried out with other metal clusters and nanoparticles and with a variety of metal chalcogenide systems.

Beside pure metal clusters and nanoparticles an interesting class of materials are metal oxides, because they have interesting magnetic and electronic properties often paired with low toxicity. Simple easy-to-synthesize oxidic compounds are silicon-based systems such as silica particles or spherosilicate clusters, therefore these systems are often used as model compounds for the class of metal oxides, although they do not really represent the class of transition metal oxides that are probably more often used in technological relevant areas. Silica particles or spherosilicate clusters both have in common that the surface contains reactive oxygen groups that can be used for further functionalization (Fig. 1.8). Mono-functional polyhedral silsesquioxane (POSS) derivatives of the type $\mathrm{R}^{\prime} \mathrm{R}_{7} \mathrm{Si}_{8} \mathrm{O}_{12}\left(\mathrm{R}^{\prime}=\right.$ functional group, $\mathrm{R}=$ nonfunctional group) are prepared by reacting the incompletely condensed molecule $\mathrm{R}_{7} \mathrm{Si}_{7} \mathrm{O}_{9}(\mathrm{OH})_{3}$ with $\mathrm{R}^{\prime} \mathrm{SiCl}_{3}$. The eighth corner of the cubic closo structure is inserted by this reaction, and a variety of functional organic groups $\mathrm{R}^{\prime}$ can be introduced, such as vinyl, allyl, styryl, norbornadienyl, 3-propyl methacrylate, etc (Fig. 1.8a). The incompletely condensed compounds $\mathrm{R}_{7} \mathrm{Si}_{7} \mathrm{O}_{9}(\mathrm{OH})_{3}$ are obtained when certain bulky groups R (e.g. cyclopentyl, cyclohexyl, tert.-butyl) prevent the formation of the closo structures from $\mathrm{RSiX}_{3}$ precursors and lead to the precipitation of open-framework POSS. These bulky substituents not only lead to open framework structures but also increase the solubility of the inorganic units in organic solvents. The closed cubic systems still show the high solubility which 
a)

$\mathrm{RSiCl}_{3}$
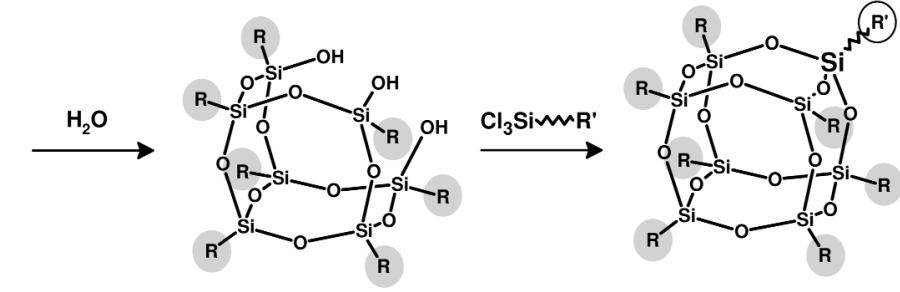

$\mathbf{R}^{\prime}=$ Reactive groups

$\mathbf{R}=$ Alkyl groups

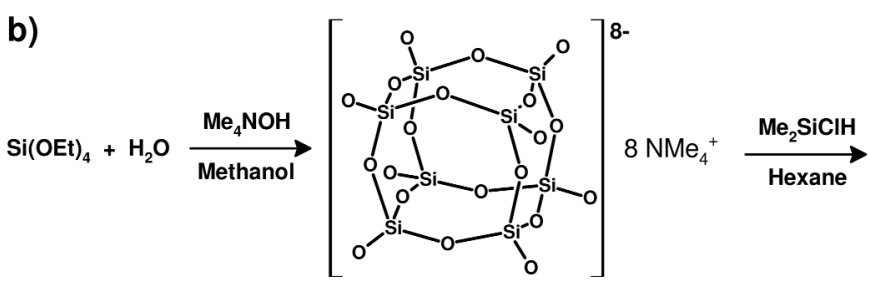

Fig. 1.8 Preparation of various well-defined silicon-based building blocks.

is necessary if the inorganic building blocks are to be incorporated in an organic environment for the functionalization of organic materials. The simple handling of these systems caused their boom in the preparation of hybrid materials. Other popular silsesquioxanes that have been prepared are the octahydrido- or the octavinyl-substituted molecules, which offer eight reactive sites. While the preparation of these systems is still very costly not least because of the low yields of the targeted products, another building block is much easier to obtain, namely spherosilicates. The polyhedral silicate species $\left[\mathrm{O}-\mathrm{SiO}_{3 / 2}\right]_{\mathrm{n}}{ }^{\mathrm{n}-}$ are commonly prepared by hydrolysis of $\mathrm{Si}(\mathrm{OR})_{4}$ in the presence of quaternary ammonium hydroxide (Fig. $1.8 \mathrm{~b})$. The best investigated compound is the cubic octamer $(\mathrm{n}=8$, "double fourring", D4R). Spherosilicates are obtained from inorganic silica sources and can be considered the smallest piece of silica. The length of a $\mathrm{Si}-\mathrm{O}-\mathrm{Si}$ edge in the D4R structure is approximately $0.3 \mathrm{~nm}$ and the diameter of the cluster ( $\mathrm{Si}-\mathrm{Si}$ distance) $0.9 \mathrm{~nm}$. Therefore the molecules can be considered sub-nanometer particles or including the organic groups - particles in the low nanometer range. The anionic oxidic surface of the species $\left[\mathrm{SiO}_{5 / 2}\right]_{\mathrm{n}}{ }^{\mathrm{n}-}$ mimics the surfaces of larger silica particles, and therefore the polyhedral silicate clusters are also model systems for (nano)particles. After functionalization usually eight reaction sites are attached to these silica cores. Some typical reactions lead to the attachment of initiating or polymerizable groups at the corners and therefore the resulting clusters can be used as multifunctional initiators for polymerizations or as crosslinking monomers.

Recently the modification and embedment of transition-metal oxide clusters and particles has become more and more important, because of their catalytic, magnetic or electric properties. Chemical approaches different to those of silicate sys- 
tems are often required for the attachment of organic groups to the surface of these compounds. The reason for these differences is the changed reactivity of these species, for example metal oxides often do not show highly nucleophilic oxygen atoms at their surface and charges are frequently delocalized over the whole cluster core. A variety of methods can be used for surface functionalization of pure clusters or nanoparticles depending on the reactivity of the surface, which often changes with parameters such as the $\mathrm{pH}$ value. In many cases only weak interactions are used to compatibilize the inorganic cluster or particle with an organic matrix, e.g. electrostatic interactions. Compatibilizing agents such as surfactants (e.g. amphiphilic molecules or block copolymers) are regularly used to increase the compatibility of the clusters or nanoparticles with an organic matrix. These molecules have two segments of which one undergoes interactions with the surface of the inorganic particle, for example by electrostatic or hydrogen bonding, and the other one, commonly a nonpolar block, interacts with the surrounding organic phase. However, functionalization of the cluster's surface by stable attachment of organic groups is preferred to a compatibilization with surfactants. Two methods can be employed for such a modification: either a cluster or particle is prepared in a first step and the surface is subsequently modified, or the surface functionalization is obtained in situ during the preparation.

Post-synthetic modification Post synthetic modification means that the cluster or nanoparticle is formed in a first step applying well-established procedures and the functionalization with organic groups is applied in a second step. Reactive surface functionalizations are required that allow a chemical reaction with the surface decorating molecules, for example nucleophilic substitution reactions. In the case of silica-based building blocks typically surface OH-groups are reacted with so-called silane coupling agents of the general composition $\mathrm{R}_{4-\mathrm{n}} \mathrm{SiX}_{\mathrm{n}}(\mathrm{n}=1-3 ; \mathrm{R}=$ functional or nonfunctional organic group; $\mathrm{X}=$ halide or $\mathrm{OR}^{\prime}$ ) to form stable covalent bonds as shown in Fig. 1.9. These molecules contain reactive $\mathrm{Si}-\mathrm{Cl}$ or $\mathrm{Si}-$ OR groups that react with surface $\mathrm{Si}-\mathrm{OH}$ groups to form stable $\mathrm{Si}-\mathrm{O}-\mathrm{Si}$ bonds. A plethora of such coupling agents is commercially available, containing various organic functional groups. If the desired group is not commercially accessible the molecule can easily be synthesized by a hydrosilation reaction. These compounds are used for the modification of silica particles or silica networks obtained in the sol-gel process. Other surface reactions can also be applied, for example the transformation of silanol groups into $\mathrm{Si}-\mathrm{Cl}$ bonds followed by reactions with nucleophiles.

Prominent examples of nanoparticles that are functionalized by such methods are so-called Stöber particles which are monodispersed silica particles with

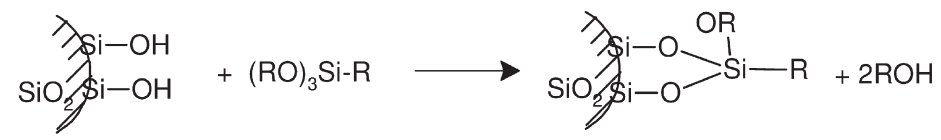

Fig. 1.9 A typical reaction of surface silanol-groups at the surface of an silica nanoparticle with a silane coupling agent. 
diameters between $5 \mathrm{~nm}$ and $200 \mathrm{~nm}$ and low particle size distributions. Similarly to spherosilicates they are formed from $\mathrm{Si}(\mathrm{OR})_{4}$ under alkaline conditions.

Only a few oxo clusters of other elements have similarly been substituted by functional silane coupling agents. The reason is that the surface functionalities in most clusters regularly lack the reactivity, e.g. basicity, of silicate surfaces, which is often based on the fact that negative charges are delocalized over the whole cluster. One of the few examples is the lacunary heterotungstate cluster $\left[\mathrm{SiW}_{11} \mathrm{O}_{39}\right]^{8-}$, in which the negative charge is rather located on two oxygen atoms. Derivatization of this cluster was obtained by reaction with various organotrichloro- or organotriethoxysilanes $\mathrm{RSiX}_{3}$ ( $\mathrm{X}=\mathrm{Cl}$ or $\mathrm{OEt}$ ) containing polymerizable groups $\mathrm{R}(\mathrm{R}=$ allyl, vinyl, styryl or 3-methacryloxypropyl, $\left.\left[\left(\mathrm{CH}_{2}\right)_{3} \mathrm{C}(\mathrm{O}) \mathrm{OCMe}=\mathrm{CH}_{2}\right]\right)$. The obtained anionic clusters have the composition $\left[\mathrm{SiW}_{11} \mathrm{O}_{35}\left(\mathrm{O}_{5} \mathrm{Si}_{2} \mathrm{R}_{2}\right)\right]^{4-}$ containing two functional organic substituents per cluster unit.

Another way to attach functional groups to the surface of a preformed building block is the exchange of surface groups similar to the above mentioned ligand exchange on gold clusters. In such a reaction the charge and coordination number balance of the surface atoms must be retained, which is more complicated in the case of metal oxides with their heterogeneous surface than with the homogeneous surface of metal clusters and particles. Electrostatic or weak coordinative bonds between surface metals and organic groups are ideally suited for this reaction type. Many stabilized metal oxide species such as titanium oxo clusters can, for example, exchange surface alkoxide groups with other alkoxides.

In the case of metal oxo clusters or metal oxide particles containing metals in high oxidation states it is often difficult to attach functionalized surface molecules, like unsaturated bonds, during their synthesis because of the strong oxidizing conditions either of the cluster itself or the oxidative reaction conditions during their synthesis. Therefore such ligand exchange reactions offer a good way to form such functionalized molecules. Organic groups can also be attached to the cluster surface by bi- or multidentate (chelating or bridging) ligands via coordinative interactions, examples for anchor groups on metal oxide clusters or particles are carboxylates, sulfonates, phosphonates, $\beta$-diketonates, etc. These groups may carry organic functionalities, such as polymerizable double bonds. However, sometimes strong ligands can lead to the reorganization of a cluster surface or even to the degradation of transition-metal clusters or a partial degradation of the particles, which has been shown in several cases.

Beside the interaction types already mentioned, ionic interactions have also been employed for surface attachment of functional groups. Knowledge of the surface charges is necessary for this method. Charges of inorganic clusters or particles are sometimes influenced by variations of the reaction environment. Metal oxides, for example, can change their surface charge over a wide range depending on the metals and the $\mathrm{pH}$ value (isoelectric point). Typical anchor groups for the interaction with such charged surfaces are carboxylates, sulfonates, phosphonates as examples for anionic groups and ammonium groups as an example for cationic groups.

A special technique for the controlled formation of hybrid materials that relates on surface charges and their interaction with counterions is the so called layer-by- 

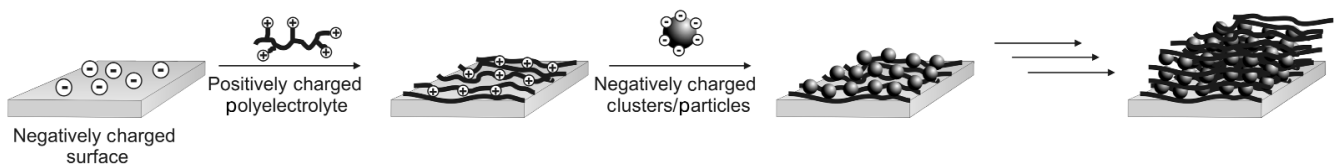

Fig. 1.10 Principle of layer-by-layer deposition.

layer (LbL) deposition. It allows the formation of inorganic-organic hybrid materials using the different charges on the surfaces of inorganic and organic polyelectrolytes. For example, anionic charges on a flat surface can be used to deposit cationically charged polyelectrolytes on it (Fig. 1.10). After deposition of the polymer the original surface charge is overcompensated and a layer of negatively charged inorganic building blocks, such as clusters or particles, can be deposited again. Afterwards again a layer of the polymer is deposited and so on. This technique enables the sequential deposition of oppositely charged building blocks. Because of its step-by-step character complex multilayer hybrid structures are readily accessible by this technique with control over layer thickness, composition and function. This method was also used for the surface functionalization of nanoparticles.

Functionalization of clusters and particles during their synthesis ("in situ functionalization") In the post-synthesis modification, functionalized building blocks are formed in two steps which are distinctly separate from each other: the inorganic core is formed first, and the functional organic groups are introduced later in a different reaction.

An alternative method is the formation of the inorganic building blocks in the presence of functional organic molecules (i.e. the functionalization of the clusters/ particles occurs in situ). This is realized in the case of metal clusters or particles if the metal core is prepared in presence of ligands that control the size of the clusters and which contain the desired functionality. One limitation is that the organic groups have to withstand the reaction conditions of the cluster core formation. For example mild reductive reactions at room temperature are usually no restriction for the majority of potential surface functionalizations, but if elevated temperatures are employed for the synthesis of the inorganic building blocks, polymerizable groups in the ligands that undergo thermal polymerization, for example (meth)acrylic or styrene systems, should be avoided. The same is valid if unsaturated, easy to oxidize bonds are present in the synthesis of metal oxo clusters or particles containing metals in high oxidation states.

Silsesquioxanes with only one substitution pattern at each silicon atom are typical examples for the in situ formation of functionalized building blocks. As mentioned above they are prepared by the hydrolysis and condensation of trialkoxy- or trichlorosilanes, thus they contain inherently one functional group that is also present in the final material. Depending on the reaction procedure either ladderlike polymers or polyhedral silsesquioxanes (POSS) are obtained. The polyhedral compounds $\left[\mathrm{RSiO}_{3 / 2}\right]_{\mathrm{n}}$ can be considered silicon oxide clusters capped by the organic groups $\mathrm{R}$. 
Polyhedral silsesquioxanes $\left[\mathrm{RSiO}_{3 / 2}\right]_{\mathrm{n}}$ are obtained by controlled hydrolytic condensation of $\mathrm{RSiX}_{3}\left(\mathrm{X}=\mathrm{Cl}, \mathrm{OR}^{\prime}\right)$ in an organic solvent. There is a high driving force for the formation of polyhedral rather than polymeric compounds, particularly if the precursor concentration in the employed solvent is low (when the concentration is increased, increasing portions of network polymers may be formed). Which oligomers are produced and at which rate, depends on the reaction conditions, such as solvent, concentration of the monomer, temperature, $\mathrm{pH}$ and the nature of the organic group R. Chlorosilanes have a higher reactivity than the corresponding alkoxysilanes. In most cases, intractable mixtures of products are obtained except for those species that precipitate from the solution.

The best investigated silsesquioxane cages are the cubic octamers, $\mathrm{R}_{8} \mathrm{Si}_{8} \mathrm{O}_{12}$. The compound $\left[\mathrm{HSiO}_{3 / 2}\right]_{8}$ is, for example, prepared by hydrolysis of $\mathrm{HSiCl}_{3}$ in a benzene / cc. $\mathrm{H}_{2} \mathrm{SO}_{4}$ mixture or by using partially hydrated $\mathrm{FeCl}_{3}$ as the water source. It is a valuable starting compound for organically substituted derivatives as the $\mathrm{Si}-\mathrm{H}$ functions of the silsesquioxane can be converted into organofunctional groups either by hydrosilation reactions (in the equation: $\mathrm{X}=\mathrm{Cl}, \mathrm{OR}^{\prime}, \mathrm{CN}$, etc.).

$$
\left[\mathrm{HSiO}_{3 / 2}\right]_{8}+8 \mathrm{XCH}_{2} \mathrm{CH}=\mathrm{CH}_{2} \rightarrow\left[\mathrm{XCH}_{2} \mathrm{CH}_{2} \mathrm{CH}_{2} \mathrm{SiO}_{3 / 2}\right]_{8}
$$

Only a few POSS with functional organic groups are insoluble enough to be obtained directly from the corresponding $\mathrm{RSiCl}_{3}$ precursor by precipitation reactions. Examples are $\left(\mathrm{CH}_{2}=\mathrm{CH}\right)_{8} \mathrm{Si}_{8} \mathrm{O}_{12}, \quad\left(p-\mathrm{ClCH}_{2} \mathrm{C}_{6} \mathrm{H}_{4}\right)_{8} \mathrm{Si}_{8} \mathrm{O}_{12}, \quad\left(\mathrm{R}_{2} \mathrm{NCH}_{2} \mathrm{CH}_{2}\right)_{8} \mathrm{Si}_{8} \mathrm{O}_{12}$ or $\left(\mathrm{ClCH}_{2} \mathrm{CH}_{2}\right)_{8} \mathrm{Si}_{8} \mathrm{O}_{12}$. These compounds can also be transformed to other functional octa(silsesquioxanes). Examples include epoxidation of vinyl groups or nucleophilic substitution of the chloro group. Nonfunctional organic groups may be converted to functional organic by standard organic reactions. For example, the phenyl groups of the cubic silsesquioxane $\mathrm{Ph}_{8} \mathrm{Si}_{8} \mathrm{O}_{12}$ were first nitrated and then reduced to give $\left(\mathrm{H}_{2} \mathrm{NC}_{6} \mathrm{H}_{4}\right)_{8} \mathrm{Si}_{8} \mathrm{O}_{12}$. The systems prepared in this way can be used as building blocks for materials depending on their functional groups.

The polyhedral compounds $\left[\mathrm{RSiO}_{3 / 2}\right]_{\mathrm{n}}$ (POSS) or $\left[\mathrm{RO}-\mathrm{SiO}_{3 / 2}\right]_{\mathrm{n}}$ discussed so far, formed by hydrolysis and condensation of a single precursor, are models for larger silica particles covered by organic groups and prepared from $\mathrm{RSi}\left(\mathrm{OR}^{\prime}\right)_{3} / \mathrm{Si}\left(\mathrm{OR}^{\prime}\right)_{4}$ mixtures. The main parameter that controls the mutual arrangement of the $\left[\mathrm{SiO}_{4}\right]$ and $\left[\mathrm{RSiO}_{3 / 2}\right]$ building blocks is the $\mathrm{pH}$. It was shown that upon sol-gel processing of $\mathrm{RSi}\left(\mathrm{OR}^{\prime}\right)_{3} / \mathrm{Si}\left(\mathrm{OR}^{\prime}\right)_{4}$ mixtures (with nonbasic groups $\mathrm{R}$ ) under basic conditions $\mathrm{Si}\left(\mathrm{OR}^{\prime}\right)_{4}$ reacts first and forms a gel network of agglomerated spherical nanoparticles. The $\mathrm{RSi}\left(\mathrm{OR}^{\prime}\right)_{3}$ precursor reacts in a later stage and condenses to the surface of the pre-formed silica nanoparticulate network. This kinetically controlled arrangement of the two building blocks from $\mathrm{RSi}\left(\mathrm{OR}^{\prime}\right)_{3} / \mathrm{Si}\left(\mathrm{OR}^{\prime}\right)_{4}$ mixtures is another method to obtain surface-modified spherical Stöber particles (see above).

In situ functionalization is also a versatile route for many transition-metal clusters. In particular the synthesis of surface-functionalized early transition metal oxo clusters was studied by in situ processes. The main differences in these reactions are that surface functionalization is included in these systems, similar to the met- 
al clusters, by functionalized ligands. For example a small zirconium oxo cluster is formed if zirconium alkoxides are mixed with functionalized carboxylic acids such as methacrylic acids. Under specific reaction conditions clusters of the type $\mathrm{Zr}_{6}(\mathrm{OH})_{4} \mathrm{O}_{4}(\mathrm{OMc})_{12}(\mathrm{OMc}=$ methacrylate $)$ are formed revealing an inorganic zirconium oxo core surrounded by a functional organic shell. The cluster core in this case, i.e. the arrangement of the eight-coordinate metal atoms and the oxygen atoms, corresponds to the basic structural unit of tetragonal zirconia. The cluster can therefore be considered the smallest possible piece of tetragonal zirconia stabilized by organic ligands. Clusters of different size and shape, and a different degree of substitution by organic ligands can be obtained by modifying the reaction conditions. The main parameters appear to be the metal alkoxide/carboxylic acid ratio and the kind of OR groups of the metal alkoxide. The surface-bound carboxylates in these clusters are loosely bound and they as well as alkoxides can be substituted by ligand exchange reactions quite easily.

Other bidentate ligands with polymerizable organic groups can be attached to the cluster surface by the in situ modification route as well (Fig. 1.11).

Interactions between metals or metal oxides cores to molecules that act as surface functionalizations are similar on a molecular scale and therefore do not usually change with the size of the core. Thus, the chemistry developed for isolated and structurally characterized metal and metal oxide clusters can also be applied for the functionalization of larger nanoparticles. This interface analogy offers the chance to study the chemistry on the molecular scale, which can be analyzed by conventional spectroscopic techniques much more easily, and transfer the obtained conclusions to the larger scale. There are many examples which show these similarities. Generally, as already mentioned above, the organic groups present in the reaction mixture and attached to the surface after the particle formation were mainly used to limit the growth of the derived particles by blocking reactive surface sites and guarantee a stable suspension in a specific solvent. Only recently have these groups been used to introduce a different surface characteristic to the surface of these building blocks or to add chemical functionalities to the surface.

Typical examples of capping agents for metals and II-VI semiconductor nanoparticles are alkylthiols for gold, CdS and related particles and tri-octyl phosphine and tri-octyl phosphine oxide for $\mathrm{CdE}(\mathrm{E}=\mathrm{S}, \mathrm{Se}, \mathrm{Te})$. Oxide nanoparticles, such as iron oxides, are often stabilized by carboxylic acids. If these systems should be incorporated into a hydrophobic matrix long alkyl chains connected to the carboxylate groups are preferred, which favors fatty acid capping agents for such
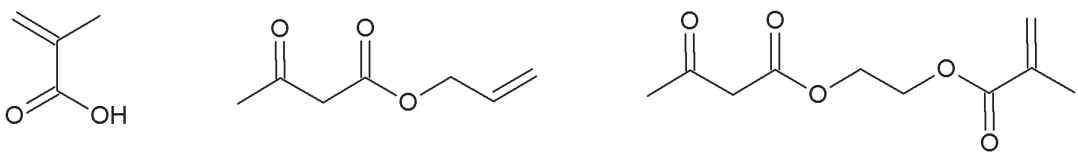

Fig. 1.11 Molecules that can act as bidentate ligands in the formation of surface-functionalized metal oxo clusters and metal oxide nanoparticles and which contain polymerizable groups for an attachment to an organic matrix. 
$\begin{aligned} & \text { Capping agent } \mathrm{HS} \\ &\left(\mathrm{CH}_{2}\right) \underset{\mathrm{n}}{\mathrm{C} \mathrm{CH}_{3}} \\ & \text { Alkylthiols }\end{aligned}$

Capped nanoparticles
$\mathrm{Ag}, \mathrm{Au}, \mathrm{CdS}$, CdSe, CdTe

$$
\begin{gathered}
\mathrm{P}\left[\left(\mathrm{CH}_{2}\right) \underset{8}{\mathrm{CH}_{3}}\right]_{3} / \mathrm{O}=\mathrm{P}\left[\left(\mathrm{CH}_{2}-\mathrm{CH}_{3}\right]_{3}\right. \\
\text { Tri-octyl phosphine (TOP) / } \\
\text { Tri-octyl phosphine oxide (TOPO) }
\end{gathered}
$$

$\mathrm{CdE}(\mathrm{E}=\mathrm{S}, \mathrm{Se}, \mathrm{Te})$

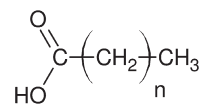

Fatty Acids

$\mathrm{Au}, \mathrm{ZnO}, \mathrm{Fe}_{3} \mathrm{O}_{4}$

Fig. 1.12 Typical capping agents used in nanoparticle

preparation changing the surface properties of the

nanoparticles to more hydrophobic.

applications. Generally attachment to the surface can occur by different interaction mechanisms and a variety of organic groups were applied for functionalization (Fig. 1.12). The selection of the capping agents is usually based on the composition of the particles and on their preparation route. The head groups of these capping agents can also be other functional molecules or polymers to graft them on the surface of the inorganic nanobuilding blocks. Mercapto-terminated linear polymers were for example used for a surface functionalization which provides a much better dispersion of the inorganic nanoparticles in various organic resins.

\subsubsection{Organic Building Blocks}

Beside the surface-functionalized inorganic building blocks described, of course organic building blocks can also be used for the formation of hybrid materials. Typical examples are oligo- and polymers as well as biological active molecules like enzymes. In principal similar methods have to be applied as in the formerly discussed example to increase the compatibility and the bonding between the two phases, therefore, a full description of the mechanisms is not necessary and would only lead to an enlargement of this introductory chapter. Hence, only a small selection of examples will be given here.

Small molecules The modification of inorganic networks with small organic molecules can be defined as the origin of hybrid materials. This is particularly true for sol-gel derived silicon-based materials. The mild conditions of the sol-gel process permit the introduction into the inorganic network of any organic molecule that consists of groups which do not interfere with these conditions, e.g. an aqueous and an acid or alkaline environment; it can then either be physically trapped in the cavities or covalently connected to the inorganic backbone (see Section 1.2.1.4). The latter is achieved by the modification of the organic molecules with hydrolysable alkoxysilane or chlorosilane groups. Phase separation is usually avoided by matching the polarity of the often hydrophobic molecules to that of the hydrophilic environment. If such a match can be obtained nearly every organic molecule can be applied to create hybrid materials. In recent years functional hybrids have been the particular focus of interest. Organic dyes, nonlinear optical groups, or switchable groups are only a small selection of molecules which have already been used to prepare hybrid materials and nanocomposites. 
Macromolecules Oligo- and polymers as well as other organic macromolecules often show different solubilities in specific solvents compared with their monomers; most often the solubility of the polymers is much lower than that of the monomers. However, many formation mechanisms for hybrid materials and nanocomposites are based on solvent chemistry, for example the sol-gel process or the wet chemistry formation of nanoparticles. Therefore, if homogeneous materials are targeted, an appropriate solvent for both the inorganic and the organic macromolecules is of great benefit. For example many macromolecules are soluble in THF which is also a reasonable good reaction environment for the sol-gel process. Additional compatibilization is obtained if the polymers contain groups that can interact with the inorganic components. Similar mechanisms of interactions can be employed as already mentioned above, i.e. groups that interact via the formation of covalent bonds or others that compatibilize between the organic and inorganic components. Some typical monomers that form either homopolymers with a good interaction or that can be used to improve the interactions by copolymerization are shown in Fig. 1.13.

A particularly interesting group of macromolecules are block copolymers, consisting of a hydrophilic and a hydrophobic segment. They can be tailored in such a way that they can react with two phases that reveal totally different chemical characters and therefore, they are known as good compatibilizers between two components. These surfactants were for example often used for the modification of nanoparticles, where one segment interacts with the surface of the particle, and the other segment sticks away from this surface. In technology such systems are often used to overcome interfaces, for example when inorganic fillers are used for the modification of organic polymers. Novel controlled polymerization methods, such as atom transfer radical polymerization (ATRP) allow the preparation of block copolymers with a plethora of functional groups and therefore novel applications will soon be available.

Particles and particle-like structures Organic colloids formed from physically or chemically crosslinked polymers can also be used as building blocks for inorganic-organic hybrid materials and nanocomposites. The good control over their properties, such as their size, the broad size range in which they can be produced, from several nanometers to micrometers, accompanied by their narrow size distribution makes them ideal building blocks for many applications. Similarly to dendrimers, special interest in these systems is achieved after their surface modification, because of their self-assembling or simply by their heterophase<smiles>C=C(C)C(=O)O</smiles><smiles>C=C(C)C(N)=O</smiles><smiles>C=C(C)C(=O)OCCO</smiles><smiles>C=C(C)C(=O)OCCN</smiles><smiles>C=C(C)C(=O)OCC[SiH3]</smiles><smiles>C=Cc1ccncc1</smiles><smiles>C=Cc1ccc(S(=O)(=O)[O-])cc1</smiles>

Fig. 1.13 Monomers that interact well with polar inorganic surfaces. 
dispersion. Many examples for the use of such colloids are mentioned in other chapters of this book (templates for porous materials, precursors for core-shell nanoparticles), so this chapter will only provide some basic insight into this topic. As already mentioned above these latex colloids are formed in aqueous dispersions which, in addition to being environmentally more acceptable or even a mandatory choice for any future development of large output applications, can provide the thermodynamic drive for self-assembling of amphiphilics, adsorption onto colloidal particles or partitioning of the hybrid's precursors between dispersed nanosized reaction loci, as in emulsion or mini-emulsion free-radical polymerizations. For the use as precursors in inorganic-organic hybrid materials styrene or acrylate homo- and copolymer core latex particles are usually modified with a reactive comonomer, such as trimethoxysilylpropyl methacrylate, to achieve efficient interfacial coupling with silica environment during the sol-gel process.

Organic colloidal building blocks were in particular used for the preparation of 3-D colloidal crystals that were subsequently applied as templates in whose voids inorganic precursors were infiltrated and reacted to inorganic materials followed by removal of the colloids. Furthermore, discrete core-shell particles can also be produced consisting of an organic core and an inorganic shell. After removal of the organic core, for example by calcination, hollow inorganic spheres are obtained.

Another type of organic macromolecular building block is the hyperbranched molecules, so-called dendrimers. Dendrimers are highly branched regular 3-D monodisperse macromolecules with a tree-like structure. These macromolecules offer a wide range of unusual physical and chemical properties mainly because they have a well-defined number of peripheral functional groups that are introduced during their synthesis as well as internal cavities (guest-host systems). In particular the deliberate control of their size and functionality makes these compounds also interesting candidates as nanoscopic building blocks for hybrid materials. Spherically shaped dendrimers are, for example, ideal templates for porous structures with porosities that are determined by the radius of the dendrimeric building block. Generally approaches for surface functionalizations of these molecules are the modification with charged end-groups or the use of reactive organic groups. Triethoxysilyl-terminated dendrimers were, for example used as precursors for micro- and mesoporous hybrid dendrimer-silica xerogels and aerogels either as single precursors or in polycondensation reactions with tetraalkoxysilanes (Fig. 1.14).

The end groups of dendrimers can also be used for an interaction with metal clusters or particles and thus nanocomposites are formed often by simply mixing the two components. For example thiol-terminated phosphorus-containing dendrimers create supramolecular assemblies with $\mathrm{Au}_{55}$ clusters. A similar route was used to substitute alkoxide groups at titanium-oxo clusters against carboxylic acid or hydroxyl end-functionalized dendrimers forming 3-D networks. Multifunctionalized inorganic molecules can also act as the core of dendrimers. POSS and spherosilicate cages were, for example, used as the core units from which dendrons were either grown divergently or to which they were appended convergently. 


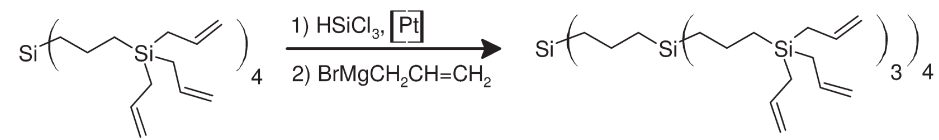

Generation 2 (G2)
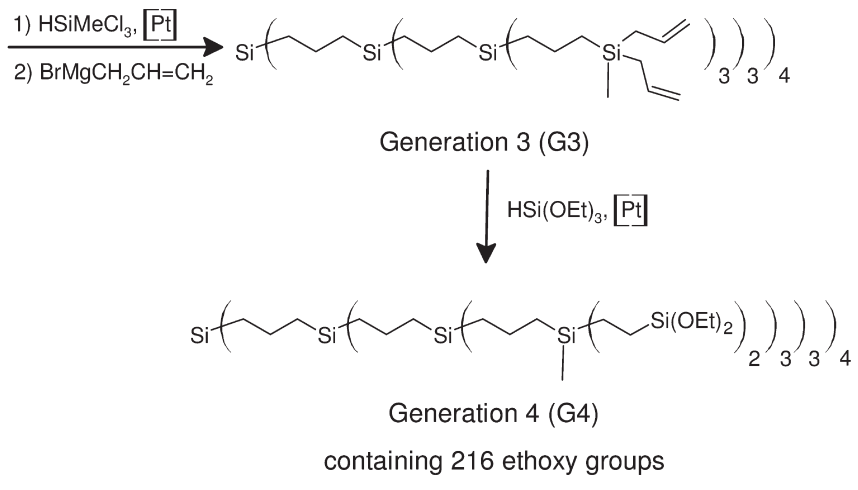

Fig. 1.14 Preparation of trialkoxysilyl-terminated carbosilane dendrimers.

Applying both silsesquioxanes and end-group functionalized dendrimers, both as multifunctional molecules crosslinked hybrid materials were obtained where welldefined inorganic molecules acted as crosslinking components.

Beside their role as crosslinking building blocks dendrimers can form hybrid materials by themselves. For example in their outer or inner shell precursors for nanoparticle synthesis can be attached to functional groups introduced during the synthesis and afterwards nanoparticles are grown within the branches of the dendrimers. One example is the complexation of metal ions in solution inside dendrimers that consist of groups, which can act as ligands, e.g. poly(amidoamine) (PAMAM) dendrimers. Subsequent chemical reactions, such as reductions, convert the entrapped metal salts to metal or semiconductor nanoparticles, which results in stable organic dendrimer-encapsulated inorganic nanoparticles.

\section{3}

\section{Structural Engineering}

An important area with respect to potential applications of hybrid materials and nanocomposites is the ability to design these materials on several length scales, from the molecular to the macroscopic scale. The importance of the design on the molecular scale was already expressed in the previous paragraphs. Because the processing of hybrid materials is more similar to that of organic polymers than to classical inorganic materials, such as ceramic or metal powders, based on the solvent-based chemistry behind the materials there is a variety of methods that can be adapted for their processing on the macroscopic scale. One has to distinguish between different applications to identify the best processing strategies. Thin films 
and coatings are generally formed by dip or spin coating, fibers are formed by spinning techniques and bulk materials are usually obtained if the often liquid precursors are simply poured into forms and curing is carried out. These techniques are used for the macroscopic processing of the materials, the molecular and microstructuring is obtained by a variety of other techniques that are mentioned below and throughout the chapters of this book.

One problem that has to be addressed in many of the macroscopic processing techniques in particular if the materials, such as the sol-gel network or the organic polymer in a hybrid are formed during the processing, is the problem of shrinkage. While in bulk materials this problem can be handled, it is more serious when films are applied on surfaces. At the interface between the coating and the support that does not follow the shrinkage considerable forces can appear that lead to inhomogeneities and cracks in the materials. As already mentioned this problem is particularly observed when the polymers or networks are formed during the processing, therefore often hybrid materials or nanocomposites are formed using preformed building blocks such as oligo- or polymers and clusters or nanoparticles to reduce this effect. However in many cases shrinkage can not totally avoided because the materials processing is usually based on solvents that are evaporated during processing.

Besides the macroscopic processing and the tailoring of the materials on a molecular scale, control of the nanometer structures is one of the major issues in the preparation of hybrid materials and nanocomposites. Many future technologies rely on hierarchically well-ordered materials from the micro- to the nanoscale. Applying the building block approach the structure of course is already engineered by the shape and distribution of the building blocks in the matrix. For example a homogeneous distribution of nanoparticles in an organic polymer has different properties than a material where the particles are agglomerated or where nanorods instead of nanoparticles are used. In addition to this building block based structural engineering the microstructure can often be influenced in hybrid materials similar to organic polymers, for example using lithographic techniques. However it is more difficult to create a structure on the nanometer level. Two different techniques can be identified for a structural control on the nanometer length scale: the top-down and the bottom-up approach. The top-down approach forms the nanostructures from larger objects by physical or chemical methods and is more engineering-related, while the bottom-up approach relies on self-organization (also called self-assembly) of molecules or nanometer sized compounds. The latter process is for many scientists the more elegant way to form large complex hierarchical structures. Think about the possibilities, for example if you have a liquid that contains your precursors and if you paint a support, it forms a hierarchical structure form the molecular over the nanometer to the macroscopic level over several hundred of square meters. This requires a detailed insight into many fundamental chemical and physico-chemical properties of the building blocks that should self assemble. In a bottom-up approach many reaction details have to be understood and controlled. Therefore, this process, although very promising, is yet only understood for quite small molecules and there are only some promising 
techniques that can already be applied for large scale technological applications. One of them is definitely the self-organization of block copolymers and their use in creating specific reaction environments. In this introductory chapter only a small selection of self-assembly mechanisms is presented, but the reader will find additional processes, explained in more detail in the following chapters.

Self-assembly is the major principle of the controlled formation of structural building blocks. It means nothing other than the spontaneous organization of unorganized systems to a complex structure, which is first of all an art to break and generate specific interactions and to work against entropy. There are several basic principles that have to be taken into account for a self-assembly process: the structure and shape of the building blocks, their interactions like the attractive and repulsive forces, their interactions with solvents, the environment where the reaction is carried out and diffusion processes. The balance between these principles is something which has to be set to create the desired structures. Two principles are employed in the synthesis of hybrid materials, the self-assembly of the building blocks of the material itself and the use of templates that can self-assemble and form a shape which is applied in the preparation of a material. Such templates are used in technology since a long time, for example as porogenes but it was only recently that they found a widespread use in the formation of hybrid materials.

Template-directed synthesis of hybrid materials Templates can fulfill various purposes, for example they can fill space and/or direct the formation of specific structures. Templates that fill space have been technologically used in materials processing for a long time; examples are porogenes for the formation of foamlike materials or single molecules that are employed in zeolite synthesis. In both examples the templates are removed after their use to form the pores in the material.

Templates for the synthesis of hybrid materials can be preformed structures such as dendrimers or nanoparticles that form 2-D or 3-D ordered structures. Furthermore the supramolecular self-organization of single molecules into larger 2-D and 3-D structures can also be employed as a template. One example is the application of amphiphilic surfactants that organize into micelles and more complicated 3-D arrangements such as hexagonal arranged rod-like structure, cubic interpenetrating networks or lamellar structures (Fig. 1.15). The latter are not

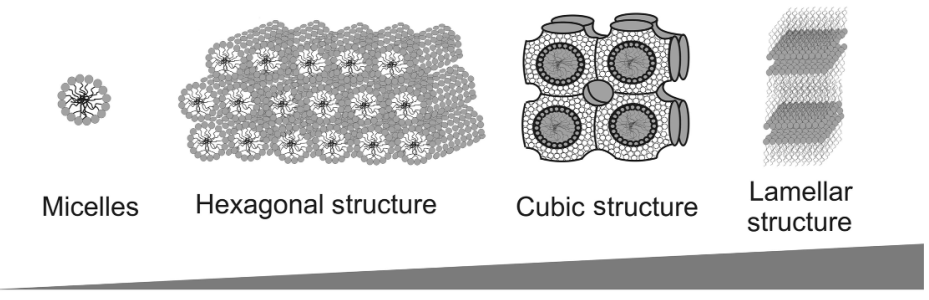

Increasing surfactant concentration

Fig. 1.15 Examples of ordered 3-D structures obtained by supramolecular self-assembly of surfactant molecules. 
usually used for structural engineering because they collapse after removal of the template. The structures formed, that are dependent on parameters such as the concentration of the surfactants in the solvent, the temperature and sometimes the $\mathrm{pH}$, consist of hydrophobic and hydrophilic regions and the interfaces between them which are applied in the formation of solid materials, primarily by sol-gel process (Fig. 1.16). As long as the organic surfactants are still incorporated in the formed inorganic matrix the materials can be considered as inorganic-organic hybrid materials. After removal of the template which commonly occurs by calcinations at temperatures above $450^{\circ} \mathrm{C}$, the materials are purely inorganic in nature. Probably the most prominent example of the use of single organic (macro)molecules and their 3-D assembly as templates for inorganic and hybrid materials is the use of surfactants in the formation of nano- and mesostructured materials porous materials (Chapter 5). Recently the silicate or metal oxide walls of the mesoporous materials were substituted by hybrid materials formed by bridged silsesquioxanes (Chapter 6) and so-called periodically mesoporous organosilicas (PMOs) were formed.

Colloids with narrow size distributions can order in 3-D objects so-called colloidal crystals. Most of the time latex or silica colloids are used for the preparation of such 3-D objects, because they can be prepared quite easily over different length scales and with the desired size distribution. They are crystallized in structurally well-ordered three-dimensional colloidal crystals that resemble the packing of atoms on a smaller scale. Because similar spheres can only fill the room by a close packing up to $74 \%$ the voids can be infiltrated by inorganic or organic reactive

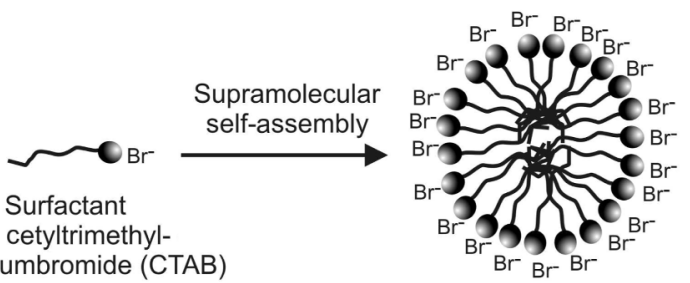
ammoniumbromide (CTAB)

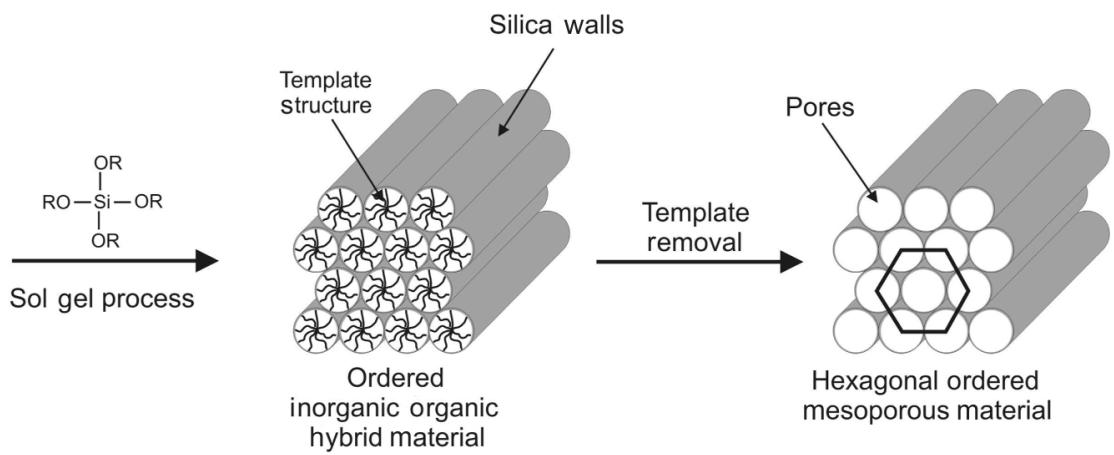

Fig. 1.16 Formation of well-ordered mesoporous materials by a templating approach. 


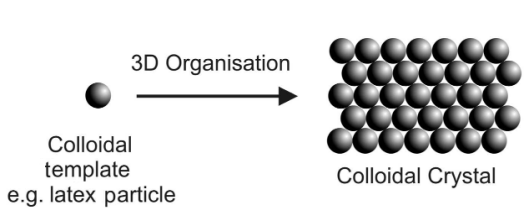

1. Infiltration with

Sol gel precursor

2. Sol gel process

Fig. 1.17 Colloidal crystal templating.

species, such as materials precursors, clusters or smaller nanoparticles and a hybrid material is formed. Generally the templates are afterwards removed and porous materials are formed (Fig. 1.17).

Many other methods can be used for the controlled preparation of nanometer structures such as soft lithography, the use of nanoscale pores, for example in anodic alumina membranes, etc. are not mentioned here and the interested reader is referred to literature in the Bibliography.

\section{4}

\section{Properties and Applications}

There is almost no limit to the combinations of inorganic and organic components in the formation of hybrid materials. Therefore materials with novel composition-property relationships can be generated that have not yet been possible. Because of the plethora of possible combinations this introductory chapter can only present some selected examples. Many of the properties and applications are dependent on the properties of the precursors and the reader is therefore referred to the following chapters.

Based on the increased importance of optical data transmission and storage, optical properties of materials play a major role in many high-tech applications. The materials used can reveal passive optical properties, which do not change by environmental excitation, or active optical properties such as photochromic (change of color during light exposure) or electrochromic (change of color if electrical current is applied) materials. Both properties can be incorporated by building blocks with the specific properties, in many cases organic compounds, which are incorporated in a matrix. Hybrid materials based on silicates prepared by the sol-gel process and such building blocks reveal many advantages compared with other types of materials because silica is transparent and if the building blocks are small enough, does not scatter light, and on the other hand organic materials are often more stable in an inorganic matrix. One of the most prominent passive features of hybrid materials already used in industry are decorative coatings obtained by the embedment of organic dyes in hybrid coatings. Another advantage of hybrid materials is the increased mechanical strength based on the inorganic structures. Scratch-resistant coatings for plastic glasses are based on this principle. One of the major advantages of hybrid materials is that it is possible to include more than one function into a material by simply incorporating a second component 
with another property into the material formulation. In the case of scratchresistant coatings, for example, additional hydrophobic or antifogging properties can be introduced. However, in many cases the precursors for hybrid materials and nanocomposites are quite expensive and therefore the preparation of bulk materials is economically not feasible. One of the advantages of hybrid materials, namely, their quite simple processing into coatings and thin films, can be one solution to this disadvantage. Applying such coatings to cheaper supports can be advantageous. Silica is preferred as the inorganic component in such applications because of its low optical loss. Other inorganic components, for example zirconia, can incorporate high refractive index properties, or titania in its rutile phase can be applied for UV absorbers. Functional organic molecules can add third order nonlinear optical (NLO) properties and conjugated polymers, conductive polymers can add interesting electrical properties. Nanocomposite based devices for electronic and optoelectronic applications include light-emitting diodes, photodiodes, solar cells, gas sensors and field effect transistors. While most of these devices can also be produced as fully organic polymer-based systems, the composites with inorganic moieties have important advantages such as the improvement of longterm stability, the improvement of electronic properties by doping with functionalized particles and the tailoring of the band gap by changing the size of the particles.

The enhancement of mechanical and thermal properties of polymers by the inclusion of inorganic moieties, especially in the form of nanocomposites, offers the possibility for these materials to substitute classical compounds based on metals or on traditional composites in the transportation industry or as fire retardant materials for construction industry.

Medical materials are also one typical application area of hybrid materials, as their mechanical properties can be tailored in combination with their biocompatibility, for example nanocomposites for dental filling materials. A high content of inorganic particles in these materials provides the necessary toughness and low shrinkage, while the organic components provide the curing properties combined with the paste-like behavior. Additional organic groups can improve the adhesion properties between the nanocomposites and the dentine.

Composite electrolyte materials for applications such as solid-state lithium batteries or supercapacitors are produced using organic-inorganic polymeric systems formed by the mixture of organic polymers and inorganic moieties prepared by sol-gel techniques. In these systems at least one of the network-forming species should contain components that allow an interaction with the conducting ions. This is often realized using organic polymers which allow an interaction with the ions, for example via coordinative or by electrostatic interactions. One typical example is proton conducting membranes which are important for the production of fuel cells The application of hybrid composites is interesting for these systems because this membrane is stable at high temperatures compared with pure organic systems.

These are only some applications for hybrid materials and there is a plethora of systems under development for future applications in various fields. 
1.5

\section{Characterization of Materials}

The range of characterization methods used in the analysis of the composition, the molecular and nanometer structure as well as the physical properties of hybrid materials is quite large. Many of these methods are specific for particular materials' compositions, therefore a complete list of these techniques is out of the range of this chapter. Here only a small selection of techniques often used for the investigation of hybrid materials is explained. Compared with classical inorganic materials, hybrids are often amorphous so well-established characterization techniques such as X-ray diffraction are limited. Many methods used are more related to the characterization of amorphous organic polymers. The heterogeneous nature of hybrid materials means that generally a variety of analytical techniques has to be used to get a satisfactory answer to structureproperty relationships.

NMR Liquid state NMR techniques are a well-known powerful tool in the characterization of solutions. The advantages of NMR spectroscopy is that it is a very sensitive technique for the chemical environment of specific nuclei and a plethora of nuclei can be investigated, which makes it also an interesting technique for solid materials. However, contrary to solution NMR where spectra usually consist of a series of very sharp lines due to an averaging of all anisotropic interactions by the molecular motion in the solution, this is different for a solid sample, where very broad peaks are observed due to anisotropic or orientation-dependent interactions. In principle high-resolution solid state NMR spectra can provide the same type of information that is available from corresponding solution NMR spectra, but special techniques/equipment are required, including magic-angle spinning (MAS), cross polarization (CP), special pulse sequences, enhanced probe electronics, etc. An additional advantage of the technique is that it is nondestructive. In this chapter we will not go into the details of this technique and the reader is referred to the corresponding literature. Here we will discuss the usefulness of the method on a selected example.

As mentioned, there are a variety of nuclei that can be used as probes in solid state NMR because of their NMR activity, many of them are also interesting for hybrid materials such as C, Si, Al, Sn, V, P, F and many others. By far the most investigated nucleus in the field of hybrid materials is ${ }^{29} \mathrm{Si}$.

${ }^{29} \mathrm{Si} \mathrm{NMR}$ is a powerful tool in the determination of the relative proportions of different silicon species in sol-gel derived materials. Therefore it offers insights into the kinetics of the process and the understanding of its fundamental parameters, such as precursor structure and reaction conditions. It is sensitive to the first and second nearest neighbors and therefore one can distinguish between different silicon atoms in the final material and their surroundings. Typical for hybrid materials is the nomenclature with letters and numbers. Four different species can be observed in hybrid materials derived by the sol-gel process depending on their substitution pattern at the silicon. In principle one can have 
one, two, three or four oxygen atoms surrounding the silicon atom and respectively the number of carbon atoms is reduced from three to zero. The abbreviations for these substitution patterns are $\mathrm{M}\left(\mathrm{C}_{3} \mathrm{SiO}\right), \mathrm{D}\left(\mathrm{C}_{2} \mathrm{SiO}_{2}\right), \mathrm{T}\left(\mathrm{CSiO}_{3}\right)$ and $\mathrm{Q}\left(\mathrm{SiO}_{4}\right)$. Depending how many $\mathrm{Si}$ atoms are connected to the oxygen atoms 4 to 1 a superscript is added to the abbreviation. In addition these silicon species have different chemical shifts which helps to distinguish between them. Some additional examples are shown in Chapter 6.

X-ray photoelectron spectroscopy (XPS) X-ray photoelectron spectroscopy (XPS) is a surface sensitive analytical tool used to examine the chemical compositions and electronic state of the surface of a sample. The sample is placed under high vacuum and is bombarded with X-rays which penetrate into the top layer of the sample ( nm) and excite electrons (referred to as photoelectrons). Some of these electrons from the upper approximately $5 \mathrm{~nm}$ are emitted from the sample and can be detected. The kinetic energy of these electrons is measured by an analyzer.

If monochromatic X-rays are used, the energy of an emitted photon imparts on an electron is a known quantity. The binding energy of the ejected electron can then be determined from:

$$
\mathrm{E}_{\text {binding }}=\mathrm{E}_{\text {photon }}-\mathrm{E}_{\text {kinetic }}-\Phi
$$

where $\Phi$ is the work function of the material.

The energy of the core electrons is very specific for the element that the atom belongs to therefore the spectrum gives information on the elemental composition of the thin surface region. Shifts in the binding energies provide additional chemical information (e.g. the oxidation state of the element). The technique also offers the opportunity to use an ion bombardment to sputter the surface layer by layer and to obtain therefore a deep profile.

Electron microscopy Electron microscopy became one of the most important techniques to characterize the materials morphology on the nanometer and nowadays even on the atomic scale. This method provides a direct image of the sample and by coupling the microscopy with analytical techniques even elemental distribution and other properties can be resolved in these dimensions. Looking at electron micrographs one has always to be aware of some limiting points: (a) all specimens are in high vacuum and probably have another shape in a liquid or gel-like surrounding, (b) the images reveal only a small fragment of the whole sample raising the possibility that the region analyzed may not be characteristic of the whole sample, and (c) the sample is treated with a high energy electron beam and it was probably changed by this beam. Points (a) and (c) can partially be avoided using special technique such as cryogenic TEM where the sample is measured at low temperatures in a frozen state. In general electron microscopy results should always be confirmed by other techniques. 
Scanning electron microscopy (SEM) Scanning electron microscopy (SEM) produces high resolution images of a sample surface. SEM images have a characteristic 3-D appearance and are therefore useful for judging the surface structure of the sample. The primary electrons coming from the source strike the surface and they are inelastically scattered by atoms in the sample. The electrons emitted are detected to produce an image. Beside the emitted electrons, X-rays are also produced by the interaction of electrons with the sample. These can be detected in a SEM equipped for energy dispersive X-ray (EDX) spectroscopy. Different detection modes can be applied such as the detection of backscattered electrons or the electron backscatter diffraction (EBSD) which gives crystallographic information about the sample.

The spatial resolution of the SEM techniques depends on various parameters, most of them are instrument related. Generally the resolution goes down to $20 \mathrm{~nm}$ to $1 \mathrm{~nm}$, which is much lower than that of transmission electron microscopy (TEM) but SEM has some advantages compared with TEM. For example a quite large area of the specimen can be imaged, bulk materials can be used as samples and, as mentioned, a variety of analytical modes is available for measuring the composition and nature of the specimen.

Transmission electron microscopy (TEM) In transmission electron microscopy (TEM) images are produced by focusing a beam of electrons onto a very thin specimen which is partially transmitted by those electrons and carries information about the inner structure of the specimen. The image is recorded by hitting a fluorescent screen, photographic plate, or light sensitive sensor such as a CCD camera. The latter has the advantage that the image may be displayed in real time on a monitor or computer.

It is often difficult in TEM to receive details of a sample because of low contrast which is based on the weak interaction with the electrons. Particularly samples that have a high content of organic components often reveal this problem, which can partially be overcome by the use of stains such as heavy metal compounds. The dense electron clouds of the heavy atoms interact strongly with the electron beam.

However, sometimes the organic components of the sample are not detected because they decompose in the electron beam; this can be avoided using cryogenic microscopy, which keeps the specimen at liquid nitrogen or liquid helium temperatures (cryo-TEM).

Similar to SEM further information about the sample can be obtained by analytical TEM, for example the elemental composition of the specimen can be determined by analyzing its X-ray spectrum or the energy-loss spectrum of the transmitted electrons. Additionally if the material observed is crystalline, diffraction patterns are obtained that give information about the crystal orientation and very powerful instruments can even investigate the crystal structure.

Modern high-resolution TEM (HRTEM) goes down to a resolution $<100 \mathrm{pm}$. 
One of the major limitations of TEM is the extensive sample preparation, which makes TEM analysis a relatively time consuming process with a low throughput of samples.

Atomic force microscopy (AFM) Atomic force microscopy (AFM) became a very important tool in the analysis of surfaces and nanoobjects in recent years. The method is intriguingly simple; a cantilever with a sharp tip at its end, typically composed of silicon or silicon nitride with tip sizes on the order of nanometers is brought into close proximity of a sample surface. The van der Waals force between the tip and the sample leads to a deflection of the cantilever. Typically the deflection is measured applying a laser beam, which is reflected from the top of the cantilever into an array of photodiodes. If the tip is scanned at constant height, there would be a risk that the tip would collide with the surface, causing damage to the sample. Therefore, in most cases a feedback mechanism is employed to adjust the tip-to-sample distance to keep the force between the tip and the sample constant. Generally, the sample is mounted on a piezoelectric holder in all three space directions ( $x$ and $y$ for scanning the sample, $z$ for maintaining a constant force). The resulting map of $s(x, y)$ represents the topography of the sample.

Primary modes of operation for an AFM are contact mode, non-contact mode, and dynamic contact mode. In the contact mode operation, the force between the tip and the surface is kept constant during scanning by maintaining a constant deflection. In the non-contact mode, the cantilever is externally oscillated at or close to its resonance frequency. The oscillation gets modified by the tip-sample interaction forces; these changes in oscillation with respect to the external reference oscillation provide information about the sample's properties. In dynamic contact mode, the cantilever is oscillated such that it comes in contact with the sample with each cycle, and then enough force is applied to detach the tip from the sample.

AFM technique has several advantages over other nanoanalysis tools, such as the electron microscope. Contrary to other methods AFM provides a 3-D surface profile of a sample. Furthermore, samples viewed by AFM do not require any special treatment such as high vacuum and it is a non destructive technique, which is not the case for electron microscope images because here high energy electron beams often destroy the organic parts of a hybrid sample.

However, there are also limits of this method; for example, AFM can only show a maximum height on the order of micrometers and a maximum area of around 100 by 100 micrometers and the scanning speed is quite low compared with SEM.

X-ray diffraction X-ray diffraction is regularly used to identify the different phases in a polycrystalline sample. Two of its most important advantages for analysis of hybrid materials are that it is fast and nondestructive. When the positions and intensities of the diffraction pattern are taken into account the pattern is unique for a single substance. The X-ray pattern is like a fingerprint and mixtures of different crystallographic phases can be easily distinguished by comparison with reference data. Usually electronic databases such as the Inorganic Crystal Structure 
Database (ICSD) are employed for this comparison. The major information one gets from this method is the crystalline composition and the phase purity. In the case of semicrystalline or amorphous materials broad humps are observed in the diffractogram. Therefore the degree of crystallinity can be qualitatively estimated.

If the crystallites of the powder are very small the peaks of the pattern will broaden. From this broadening it is possible to determine an average crystallite size by the Debye-Scherrer equation:

$$
d=\frac{k \lambda}{B \cos \theta}
$$

where $k$ is a factor which is usually set to $0.9, \lambda$ is the wavelength of the X-ray radiation, $\mathrm{B}$ is the broadening of the diffraction line measured at half of its maximum intensity (radians) and $\theta$ is the Bragg angle. An error for the crystallite size by this fomular can be up to $50 \%$ which is appropriate.

Small-angle X-ray scattering (SAXS) SAXS is a technique where the source for the scattering of the X-rays is the inhomogeneities in the sample within the nanometer range. SAXS patterns are recorded at very low angles (typically $<3-5^{\circ}$ ). In this angular range, information about the shape and size of the inhomogeneities, which can be, for example, clusters or nanoparticles in an organic matrix, and their distances is obtained. Furthermore pores can also be defined as inhomogeneities therefore SAXS is also used to characterized regularly ordered porous materials. The resolution of SAXS experiments is strongly dependent on the equipment. The highest resolution is obtained at beamlines at synchrotrons.

Thermal analysis techniques Thermogravimetric analysis (TGA) studies the weight changes of samples in relation to changes in temperature. TGA is commonly employed with respect to hybrid materials and nanocomposites to investigate the thermal stability (degradation temperatures), the amount of inorganic component, which usually stays until the end of the measurement due to its high thermal resistance, and the level of absorbed moisture or organic volatiles in these materials. Typically TGA plots show the weight lost in relation to the temperature and typical ranges that can be distinguished are the lost of moisture and absorbed solvents up to $150^{\circ} \mathrm{C}$, the decomposition of organic components between 300 and $500^{\circ} \mathrm{C}$. Usually the measurements are carried out under air or an inert gas.

Differential scanning calorimetry (DSC) is a thermoanalytical technique that compares the difference in the amount of heat required to increase the temperature of a sample and a reference with a well-defined heat capacity measured as a function of temperature. Both the sample and the reference are maintained at the same temperature throughout the experiment. Generally, the temperature program for a DSC analysis is designed such that the sample holder temperature increases linearly as a function of time. The basic principle underlying this technique is that, when the sample undergoes a physical transformation such as a 
phase transition or thermal decomposition, more or less heat is required compared with the reference, to maintain both at the same temperature. Whether more or less heat must flow to the sample depends on whether the process is exothermic or endothermic. Melting points of solids, for example, require more heat flow to the sample therefore these processes are endothermic, while thermal decompositions, for example oxidation processes are mostly exothermic events. An exothermic or endothermic event in the sample results in a deviation in the difference between the two heat flows to the reference and results in a peak in the DSC curve (plot of heat flow against temperature). The difference in heat flow between the sample and reference also delivers the quantitative amount of energy absorbed or released during such transitions. This information can be obtained by integrating the peak and comparing it with a given transition of a known sample.

Similar to TGA the experiments can be carried out under oxygen and other atmospheres such as inert gas.

\section{6}

\section{Summary}

Hybrid materials represent one of the most fascinating developments in materials chemistry in recent years. The tremendous possibilities of combination of different properties in one material initiated an explosion of ideas about potential materials and applications. However, the basic science is sometimes still not understood, therefore investigations in this field in particular to understand the structure-property relationships are crucial. This introductory chapter is intended to give an overview of critical issues in the synthesis and to guide the reader to the other chapters in this book, which focus on more specialized topics. This introduction has shown the importance of the interface between the inorganic and organic materials which has to be tailored to overcome serious problems in the preparation of hybrid materials. Different building blocks and approaches can be used for their preparation and these have to be adapted to bridge the differences of inorganic and organic materials. Beside the preparation of hybrid materials, their nano- and microstructure formation, processing and analysis is important. A variety of techniques can be used for these issues and several of them have been introduced to the reader.

\section{Bibliography}

\section{Sol-gel process}

J. D. Wright, N. Sommerdijk, Sol-Gel Materials: Their Chemistry and Biological Properties, Taylor \& Francis Group, London, 2000.

U. Schubert, N. Hüsing, A. Lorenz, Hybrid Inorganic-Organic Materials by Sol-Gel
Processing of Organofunctional Metal Alkoxides. Chem. Mater. 1995, 7, 2010-2027.

C. J. Brinker, G. W. Scherer, Sol-Gel Science: The Physics and Chemistry of Sol-Gel Processing, Academic Press, London, 1990. 


\section{Hybrid materials}

Special issue of J. Mater. Chem. 2005, 15, 3543-3986.

P. Gómez-Romero, C. Sanchez (Eds.) Functional Hybrid Materials, Wiley-VCH, Weinheim, 2004.

G. Schottner, Hybrid Sol-Gel-Derived Polymers: Applications of Multifunctional Materials. Chem. Mater. 2001, 13, 3422-3435.

G. Kickelbick, U. Schubert, Inorganic Clusters in Organic Polymers and the Use of Polyfunctional Inorganic Compounds as Polymerization Initiators, Monatsh. Chem. 2001, 132, 13.

K.-H. Haas, Hybrid inorganic-organic polymers based on organically modified Si-alkoxides. Adv. Eng. Mater. 2000, 2, 571-582.

C. Sanchez, F. Ribot, B. Lebeau, Molecular design of hybrid organic-inorganic nanocomposites synthesized via sol-gel chemistry. J. Mater. Chem. 1999, 9, 35-44.

K. G. Sharp, Inorganic/organic hybrid materials. Adv. Mater. 1998, 10, 1243-1248.

\section{Nanocomposites}

Special issue of J. Nanosci. and Nanotechn. 2006, 6, 265-572.

R. M. Laine, Nanobuilding blocks based on the [OSiO1.5]x ( $x=6,8,10)$ octasilsesquioxanes. J. Mater. Chem. 2005, 15, 3725-3744.

A. Usuki, N. Hasegawa, M. Kato, Polyme-clay nanocomposites. Adv. Polym. Sci. 2005, 179, 135-195

B. C. Sih, M. O. Wolf, Metal nanoparticleconjugated polymer nanocomposites. Chem. Commun. 2005, 3375-3384.

C. Sanchez, B. Julian, P. Belleville, M. Popall, Applications of hybrid organic-inorganic nanocomposites. J. Mater. Chem. 2005, 15, 3559-3592.

P. Gómez-Romero, K. Cuentas-Gallegos, M. Lira-Cantu, N. Casan-Pastor, Hybrid nanocomposite materials for energy storage and conversion applications. J. Mater. Sci. 2005, 40, 1423-1428.

G. Kickelbick, Concepts for Incorporation of Inorganic Building Blocks into Organic Polymers on a Nanoscale. Progr. Polym. Sci. 2003, 28, 83-114.

Special issue of Chem. Mater. 2001, 13, 3059-3910.

\section{Porous materials}

A. Sayari, S. Hamoudi, Periodic Mesoporous Silica-Based Organic-Inorganic

\section{Bibliography}

\section{7}

Nanocomposite Materials. Chem. Mater. 2001, 13, 3151-3168.

F. Hoffmann, M. Cornelius, J. Morell, M. Fröba, J. Nanosci. Nanotechnol. 2006, 6, 265-288.

B. Hatton, K. Landskron, W. Whitnall, D. Perovic, G. A. Ozin, Past, Present, and Future of Periodic Mesoporous Organosilicas The PMOs. Acc. Chem. Res. 2005, 38, 305-312

J-L. Shi, Z.-L. Hua, L.-X. Zhang, Nanocomposites from ordered mesoporous materials. J. Mater. Chem. 2004, 14, 795-806.

R. Gangopadhyay, A. De, Conducting Polymer Nanocomposites: A Brief Overview. Chem. Mater. 2000, 12, 608-622.

C. J. Brinker, Y. Lu, A. Sellinger, H. Fan, Evaporation-induced self-assembly.

Nanostructures made easy. Adv. Mater. 1999, 11, 579-585.

N. Hüsing, U. Schubert, Aerogels - airy materials: chemistry, structure, and properties. Angew. Chem. Int. Ed. 1998, 37, 22-45.

\section{Building blocks \\ Macromolecules}

D. Y. Godovsky, Device applications of polymernanocomposites. Adv. Polym. Sci. 2000, 153, 163-205.

J. Wen, G. L. Wilkes, Garth L.

Organic/Inorganic Hybrid Network Materials by the Sol-Gel Approach. Chem. Mater. 1996, 8, 1667-1681.

\section{Dendrimers}

G. J. de A. A. Soler-Illia, L. Rozes, M. K. Boggiano, C. Sanchez, C.-O. Turrin, A.-M. Caminade, J.-P. Majoral, New mesotextured hybrid materials made from assemblies of dendrimers and titanium(IV)-oxo-organo clusters. Angew. Chem. Int. Ed. 2000, 39, 4249-4254.

J. L. Hedrick, T. Magbitang, E. F. Connor, T. Glauser, W. Volksen, C. J. Hawker, V. Y. Lee, R. D. Miller, Application of complex macromolecular architectures for advanced microelectronic materials. Chem. - Europ. J. 2002, 8, 3308-3319.

J. W. Kriesel, T. D. Tilley, Carbosilane dendrimers as nanoscopic building blocks for hybrid organic-inorganic materials and catalyst supports. Adv. Mater. 2001, 13, 1645-1648. 
P. R. Dvornic, C. Hartmann-Thompson, S. E. Keinath, E. J. Hill, Organic-Inorganic Polyamidoamine (PAMAM) DendrimerPolyhedral Oligosilsesquioxane (POSS) Nanohybrids. Macromolecules 2004, 37, 7818-7831.

R. W. J. Scott, O. M. Wilson, R. M. Crooks, Synthesis, Characterization, and Applications of Dendrimer-Encapsulated Nanoparticles. J. Phys. Chem. B 2005, 109, 692-704.

\section{Particles}

V. Castelvetro, C. De Vita, Cinzia.

Nanostructured hybrid materials from aqueous polymer dispersions. Adv. Coll. Interf. Sci. 2004, 108-109, 167-185.
E. Bourgeat-Lami, Organic/inorganic nanocomposite colloids in Encyclopedia of Nanoscience and Nanotechnology (Ed. H. S. Nalwa), American Scientific Publishers, Stevenson Ranch 2004.

G. Kickelbick, L. M. Liz-Marzán, Core-Shell Nanoparticles in Encyclopedia of Nanoscience and Nanotechnology (Ed. H. S. Nalwa), American Scientific Publishers, Stevenson Ranch, 2004.

\section{Engineering of nanomaterials}

G. A. Ozin, A. C. Arsenault, Nanochemistry A Chemical Approach to Nanomaterials, RSC Publishing, London, 2005. 